\title{
Relaxation of the curve shortening flow via the parabolic Ginzburg -Landau equation
}

\author{
Mariel Sáez Trumper
}

Received: 3 April 2007 / Accepted: 18 May 2007 / Published online: 29 June 2007

C) Springer-Verlag 2007

\begin{abstract}
In this paper we study how to find solutions $u_{\epsilon}$ to the parabolic Ginzburg-Landau equation that as $\epsilon \rightarrow 0$ have as interface a given curve that evolves under curve shortening flow. Moreover, for compact embedded curves we find a uniform profile for the solution $u_{\epsilon}$ up the extinction time of the curve. We show that after the extinction time the solution converges uniformly to a constant.
\end{abstract}

Mathematics Subject Classification (2000) $\quad 53 \mathrm{C} 44 \cdot 35 \mathrm{~K} 65$

\section{Introduction}

This paper concentrates in studying the relationship between the curve shortening flow and the parabolic Ginzburg-Landau equation.

Let us recall that a curve $\Gamma(x, t)$ on $\mathbb{R}^{2}$ evolves under curve shortening flow, if it satisfies the following equation:

$$
\frac{\partial \Gamma}{\partial t}=k_{\Gamma} v
$$

where $k_{\Gamma}(\theta, t)$ is the curvature of $\Gamma$ with respect to the space variable and $v$ is the unit normal vector to $\Gamma$. Moreover, notice that any smooth reparametrization of $\Gamma$ satisfies

$$
\frac{\partial \Gamma}{\partial t} \cdot v=k_{\Gamma}
$$

The behavior of regular curves under this flow has been widely studied; see for example [10-13]. However, the current understanding is much more limited in some less regular situations, as in the case of networks of curves flowing under the curve shortening flow. Important progress in this direction was made by Mantegazza et al. [16]. One of the goals of this paper is to develop a method that allow us to extend these results. 
Mean curvature flow has been related to the solution to a parabolic equation, known as parabolic Ginzburg-Landau equation, that depends on a parameter $\epsilon$. We can describe this equation as follows: let $\Omega$ be an open subset of an $m$-dimensional manifold $M$. A function $u_{\epsilon}: \Omega \times \mathbb{R}_{+} \rightarrow \mathbb{R}$ satisfies the parabolic Ginzburg-Landau equation if

$$
\begin{gathered}
\frac{\partial u_{\epsilon}}{\partial t}-\Delta u_{\epsilon}+\frac{W^{\prime}\left(u_{\epsilon}\right)}{2 \epsilon^{2}}=0 \\
u_{\epsilon}(x, 0)=\xi_{\epsilon}(x),
\end{gathered}
$$

where $W$ is a nonnegative potential whose minimum value is 0 . In some contexts in the literature this equation is also referred as the Allen-Cahn equation.

Many people have studied the behavior solutions $u_{\epsilon}$ as $\epsilon$ approaches 0 , in particular when $u_{\epsilon}$ does not depend on time, so that (3) reduces to the elliptic equation

$$
-\Delta u_{\epsilon}+\frac{W^{\prime}\left(u_{\epsilon}\right)}{2 \epsilon^{2}}=0 .
$$

Less is known about the parabolic equation. When $W$ is a potential with two minimizers Ilmanen [15] showed for a solution $u_{\epsilon}: \mathbb{R}^{n} \times[0, T] \rightarrow \mathbb{R}$ to (3) the measures $d \mu_{t}^{\epsilon} \equiv$ $\left(\frac{\epsilon}{2}\left|D u_{\epsilon}\right|^{2}+\frac{1}{\epsilon} W\left(u_{\epsilon}\right)\right) d x$ converge to Brakke's motion of varifolds by mean curvature. For a rigorous definition of this concept see [4]. This implies that if the limiting interface is "thin," then it evolves by the standard mean curvature flow. Similar results were proven earlier in a slightly less general setting in [2,7-9].

Let us define for each $t \in[0, T)$ the interface set at time $t, I(t)$, to be the set of those $x \in \mathbb{R}^{2}$ such that $u_{\epsilon}(x, t)$ does not converge to a point where $W$ attains its minimum as $\epsilon \rightarrow 0$.

Pacard and Ritoré [18] proved that certain minimal hypersurfaces, which correspond to the stationary points of the mean curvature flow, can be realized as interface sets of solutions to the elliptic Ginzburg-Landau equation. Results in this direction were also proven by Paolini [19] and by Hutchinson and Tonegawa [14]. In this paper an analogous result for the parabolic Ginzburg-Landau equation is proved. More precisely we prove

Theorem 1.1 Let $W: \mathbb{R} \rightarrow \mathbb{R}$ be a positive function, which achieves its minimum value 0 only at 1 and -1 . Consider $u_{*}$ to be a solution to the following equation:

$$
\begin{gathered}
-\left(u_{*}\right)_{x x}+\frac{1}{2} W^{\prime}\left(u_{*}\right)=0 \\
u_{*}(0)=0 \quad \text { and } \quad \lim _{x \rightarrow \pm \infty} u_{*}(x)= \pm 1 .
\end{gathered}
$$

Suppose that $\Gamma(\lambda, t)$, with space parameter $\lambda \in[0, \bar{\lambda}]$, is a smooth embedded curve in $\mathbb{R}^{2}$ (either compact or non-compact) that satisfies (1), $d(x, t)$ is the signed distance to $\Gamma$ at time $t$ and $T$ the time when the first singularity occurs.

Consider an initial condition $\psi_{\epsilon}(x)$ that satisfies

$$
\psi_{\epsilon}(x)=u_{*}\left(\frac{d(x, 0)}{\epsilon}\right) \quad \text { for } x \in V^{\prime},
$$

and

$$
\left|\psi_{\epsilon}(x)\right|=1 \quad \text { for } x \in V^{c}
$$


for tubular neighborhoods $V^{\prime} \subset V$ of $\Gamma(\lambda, 0)$. If $\Gamma(\lambda, 0)$ is not compact we also require

$$
\inf _{x, y \in \Gamma(\lambda, 0)} \operatorname{dist}(x, y) \geq \delta>0 .
$$

Moreover, we assume for non-compact curves that the tubular neighborhood $V$, where $d(x, t)$ is well defined and smooth, exists.

Let $u_{\epsilon}(x, t)$ be a solution of (3)-(4). For every $T>\bar{T} \geq 0$ there are tubularneighborhoods $U^{\prime} \subset U$ of $\Gamma(\lambda, t)$ and there is a function $-1 \leq v_{\epsilon}(x, t) \leq 1$ that satisfies

$$
\begin{gathered}
v_{\epsilon}(x, t)=u_{*}\left(\frac{d(x, t)}{\epsilon}\right) \quad \text { for } x \in U^{\prime}, \\
\left|v_{\epsilon}(x, t)\right|=1 \quad \text { for } x \in U^{c}
\end{gathered}
$$

such that

$$
\lim _{\epsilon \rightarrow 0} \sup _{(x, t) \in \mathbb{R}^{2} \times[0, \bar{T}]}\left|u_{\epsilon}(x, t)-v_{\epsilon}(x, t)\right|=0 .
$$

Remark 1.1 Theorem 1.1 shows that any embedded curve $\Gamma$ evolving under curve shortening flow can be attained, before its collapsing time, as an interface of some solution $u_{\epsilon}$ to (3). Moreover the convergence profile of this solution is given by (8)-(9).

We would like to remark that the curves considered in Theorem 1.1 can be compact or non-compact. Notice that for non-compact curves condition (7) corresponds to $\Gamma(\lambda, 0)$ being "embedded at infinity", that is $\Gamma(\lambda, 0)$ does not intersect itself at infinity. By standard maximum principle arguments, it is easy to see that this condition is preserved in time.

Remark 1.2 The proof of Theorem 1.1 can be easily generalized to the following settings:

- Theorem 1.1 holds when $u: \mathbb{R}^{n} \rightarrow \mathbb{R}$ and $\Gamma$ is a compact embedded hyper-surface in $\mathbb{R}^{n}$ evolving under mean curvature flow. Since Theorem 1.2 cannot be generalized to this setting, we will remain in the context of curves.

- Consider $u_{\epsilon}: \mathbb{R}^{2} \rightarrow \mathbb{R}^{2}$ is a vector valued solution to (3)-(4) and $W: \mathbb{R}^{2} \rightarrow \mathbb{R}$ is a positive potential with two vector-valued minima, $a$ and $b$. Then Theorem 1.1 generalizes to this context by replacing the function $u_{*}$ defined by (5)-(6) for the vector-valued solution $\gamma_{a b}: \mathbb{R} \rightarrow \mathbb{R}^{2}$ to (5), that decays exponentially to $a$ and $b$ when $x \rightarrow \pm \infty$ respectively. The existence of such a solution was proved by Sternberg in [21]. In order to prove existence of this solution some further assumptions over $W$ are necessary (see [21]). The proof of this generalization is identically to the one presented in this paper, with the only exception of Theorem 2.1, that needs to be generalized to this context. This generalization can be found in [20]. For simplicity we will prove here Theorem 1.1 as stated above.

We also prove an extension of this result:

Theorem 1.2 Let $\Gamma(\lambda, t)$ be an embedded closed compact curve satisfying (1) and $T$ its maximal time of existence. Define $x_{0}=\Gamma(\cdot, T)$, the point where $\Gamma$ collapses at time $T$.

Consider $u_{\epsilon}(x, t)$ be the solution to equations (3)-(4), for the initial condition $\psi_{\epsilon}$ given by Theorem 1.1.

Consider $p>0$ and $\eta \in C_{0}^{\infty}(\mathbb{R} \times \mathbb{R})$ such that $0 \leq \eta \leq 1$ and

$$
\eta(x, y)= \begin{cases}1, & \text { if }|x| \leq 1 \text { and }|y| \leq 1 \\ 0 . & \text { if }|x| \geq 2 \text { or }|y| \geq 2\end{cases}
$$


Define

$$
\begin{aligned}
\mathcal{A}(r, t, \epsilon)= & \left(1-\eta\left(\frac{\left|x-x_{0}\right|}{\epsilon \sqrt{2(T-t)}}, 2 \frac{T-t}{3 \epsilon^{2}|\ln \epsilon|^{3+p}}\right)\right)\left(d(x, t)+\frac{\epsilon^{2}|\ln \epsilon|^{3+p}}{\sqrt{2(T-t)}}\right) \\
& +\eta\left(\frac{\left|x-x_{0}\right|}{\epsilon \sqrt{2(T-t)}}, 2 \frac{T-t}{3 \epsilon^{2}|\ln \epsilon|^{3+p}}\right)\left(-\sqrt{2(T-t)}+\frac{\epsilon^{2}|\ln \epsilon|^{3+p}}{\sqrt{2(T-t)}}\right) .
\end{aligned}
$$

Then there exist neighborhoods $U^{\prime} \subset U$ of $\Gamma(\lambda, t)$ and function $v_{\epsilon}^{*}(x, t)$ such that:

$$
\begin{gathered}
v_{\epsilon}^{*}(x, t)=u_{*}\left(\frac{\mathcal{A}(x, t, \epsilon)}{\epsilon}\right) \quad \text { for } x \in U^{\prime} \\
\left|v_{\epsilon}^{*}(x, t)\right|=1 \quad \text { for } x \in U^{c} \text {, and } \\
\lim _{\epsilon \rightarrow 0} \sup _{(x, t) \in \mathbb{R}^{2} \times[0, \infty)}\left|u_{\epsilon}(x, t)-v_{\epsilon}^{*}(x, t)\right|=0 .
\end{gathered}
$$

Remark 1.3 Theorem 1.2 shows the profile near the extinction time. Moreover, it shows that for every time $t \geq T$, the solution $u_{\epsilon}(x, t) \rightarrow 1$ uniformly.

The extinction profile given by Theorem 1.2 comes from an explicit computation in the case of a circle evolving under curve shortening flow. This computation is presented in Appendix. Due to Gage and Hamilton's result in [10] we know that near the extinction time any compact curve evolving under curve shortening flow approaches the circle described by Appendix. Combining both results we obtain the profile given by Theorem 1.2. Further geometric intuition in the circle case will be discussed in Appendix.

The author expects this approach to generalize to more general settings of shortening flow, such as networks. These networks are defined as families of $n$ regular curves $\Gamma^{i}:[0,1] \rightarrow \Omega$ that may intersect each other or self-intersect only at their endpoints. A network is said to be flowing by curve shortening flow if the interior of each constituent curve evolves in time according to this flow. Mantegazza et al. [16] focused on the simplest kind networks, called triods, that consist only of three curves. They proved existence and studied regularity results for this system, but they impose a strong constraint, namely that the angles between the tangents at the meeting point be $120^{\circ}$ for all $t \in[0, T)$. Formal computations of Bronsard and Reitich in [5] show that this condition would be determined by the function $W$ when the flow is regarded as the interface set of a solution to an appropriate Ginzburg-Landau equation. We also expect that other information about the triod flow will follow by considering it as an interface set of solution $u_{\epsilon}$ to (3). The main technical difficulty to extend the result to this case relies in the fact that is necessary to consider vector valued solution $u_{\epsilon}: \mathbb{R}^{2} \rightarrow \mathbb{R}^{2}$ to the equation (11)-(12), as well as a potential $W: \mathbb{R}^{2} \rightarrow \mathbb{R}$ with several minima. For such a potential is not even clear the existence of profiles analogous to (5)-(6) that decay at infinity to more than two minima. It is possible that other generalizations and results for Mean Curvature Flow might be derived from this approach. For example, an analogous theorem to Theorem 1.2 could provide information about the formation of singularities of Mean Curvature Flow for dimensions greater that 3. In general, since for every $\epsilon>0$, Eq. (3) is strictly parabolic for every $t>0$ and does not develop singularities in finite time (in contrast with the Mean Curvature Flow equation), this approach provides us with a relaxation of Mean Curvature Flow, that agrees with Brakke flow in the context discussed by Ilmanen in [15]; and, moreover, can be studied in the frame work of parabolic differential equations. 
After concluding this paper the author learned about results similar to Theorem 1.1 in [2,3,7-9], among others . The proofs in these papers rely strongly on the use of maximum principle, hence do not generalize to the case of a vector valued Ginzburg-Landau equation. Most of the results here extend easily to the vector valued case. The generalizations will be pointed out along this paper. In a forthcoming paper we will treat the case of vector valued Ginzburg-Landau equation with a potential $W$ with three minima.

We would also would like to contrast the profile obtained in Theorem 1.2 with the results in [6], where Buttá obtains a similar profile for a nonlocal version of a related equation. We would like to remark that Theorem 1.2 was developed independently of Buttá's paper. It is also possible to find a numerical algorithm that explores developement of singularities in [17].

We organize the paper in the following way: in Sect. 2 we show general estimates and constructions, that will be used in the coming sections. Section 3 deals with proving existence of solutions to the Eq. (3) and finding estimates over these solutions. Finally, Sect. 4 contains the proofs of Theorems 1.1 and 1.2. As mentioned above, in the proof of Theorem 1.2 the particular example of a circle will be used. The proof of this particular example can be found in Appendix.

The results presented in this paper are partially contained in [20]. I would like to acknowledge my adviser Rafe Mazzeo for his help and support. I would also like to thank Giovanni Bellettini for pointing out relevant references.

\section{Previous results}

In this section we present a collection of a priori estimates and constructions that will be essential in the proof of Theorems 1.1 and 1.2. We remark that most of the estimates presented here and in Sect. 3 can be easily generalized for vector valued solution $u_{\epsilon}$.

We first establish some notation. Let

$$
P u=\frac{\partial u}{\partial t}-\Delta u \text {. }
$$

Then we can write (3)-(4) as

$$
\begin{gathered}
P u_{\epsilon}+\frac{1}{2 \epsilon^{2}} W^{\prime}\left(u_{\epsilon}\right)=0 \\
u_{\epsilon}(x, 0)=\xi_{\epsilon}(x)
\end{gathered}
$$

Let us start by proving some a priori bounds for solutions to (11)-(12).

Theorem 2.1 Let $u_{\epsilon}(x, t)$ satisfies (11)-(12) in $\mathbb{R}^{2} \times[0, T]$ (with $T$ possibly equal to infinity), where $W \in C^{2}$ is bounded below, has a finite number of critical points $\left\{a_{i}\right\}_{i=1}^{n}$ and is such that $W(v) \rightarrow \infty$ as $|v| \rightarrow \infty$. We also assume that there is a $\delta>0$ such that $\left|W^{\prime}(v)\right| \geq \delta$ as $|v| \rightarrow \infty$. If $u_{\epsilon}(x, 0)=\xi_{\epsilon}(x)$ is bounded and for every $t \geq 0$ holds $W^{\prime}\left(u_{\epsilon}\right)(x, t) \rightarrow 0$ as $|x| \rightarrow \infty$, we have that $\sup _{\mathbb{R}^{2} \times[0, T]}\left|u_{\epsilon}(x, t)\right| \leq C$, where the constant $C$ only depends on $\xi_{\epsilon}$ and $W\left(a_{i}\right)$.

Since $W$ is bounded below and proper this theorem is a corollary of the following lemma.

Lemma 2.1 Let $u$ be a solution to (11)-(12) that satisfies $W^{\prime}\left(u_{\epsilon}\right)(x, t) \rightarrow 0$ as $|x| \rightarrow \infty$ for every $t$. Suppose that $W$ satisfies the hypothesis of Theorem 2.1. Then for $t \in[0, \bar{t}]$ we have $\left|W\left(u_{\epsilon}\right)(x, t)\right| \leq \max \left\{W\left(\xi_{\epsilon}\right), W\left(a_{i}\right)\right\}$ where $a_{i}$ are the critical points of $W$. 
Proof To simplify the notation we will drop the indices $\epsilon$ along this proof. Consider $v(x, t)=$ $W(u)(x, t)$; then

$$
\begin{aligned}
v_{t}-\Delta v & =W^{\prime}(u) u_{t}-\sum_{i}\left(W^{\prime}(u) u_{x_{i}}\right)_{x_{i}} \\
& =W^{\prime}(u) u_{t}-W^{\prime \prime}(u)|\nabla u|^{2}-W^{\prime}(u) \Delta u
\end{aligned}
$$

Since $u$ satisfies (11), this becomes

$$
v_{t}-\Delta v+\frac{\left|W^{\prime}(u)\right|^{2}}{2 \epsilon^{2}}+W^{\prime \prime}(u)|\nabla u|^{2}=0
$$

Suppose that $v$ has a maximum at $\left(x_{0}, t_{0}\right)$. Then $\nabla v\left(x_{0}, t_{0}\right)=W^{\prime}(u) \nabla u\left(x_{0}, t_{0}\right)=0$, so there are two possibilities:

1. $W^{\prime}(u)\left(x_{0}, t_{0}\right)=0$ : In this case, $u\left(x_{0}, t_{0}\right)=a_{i}$ for some $i$, therefore $W(u)(x, t) \leq W\left(a_{i}\right)$ and the result of the lemma follows or

2. $\nabla u\left(x_{0}, t_{0}\right)=0$ : Since $\left(x_{0}, t_{0}\right)$ is a maximum for $v$, it is also true that $v_{t}\left(x_{0}, t_{0}\right) \geq 0$ and $\Delta v\left(x_{0}, t_{0}\right) \leq 0$. We can assume also that $W^{\prime}(u)\left(x_{0}, t_{0}\right) \neq 0$, therefore

$$
v_{t}-\Delta v+\frac{\left|W^{\prime}(u)\right|^{2}}{\epsilon^{2}}+W^{\prime \prime}(u)|\nabla u|^{2}>0
$$

which contradicts (13).

Therefore the only possible interior maxima of $v$ are critical points of $W$.

We still need to analyze the behavior of $v$ at infinity. We will show that

$$
\lim _{|x| \rightarrow \infty}|v(x, t)| \leq \max _{i} W\left(a_{i}\right) .
$$

Recall that $W^{\prime}\left(u_{\epsilon}\right)(x, t) \rightarrow 0$ as $|x| \rightarrow \infty$. In order to prove (14), we show that necessarily for any subsequence $x_{n}$ such that $\left|x_{n}\right| \rightarrow \infty$ there is an $i$ such that $u_{\epsilon}\left(x_{n}, t\right) \rightarrow a_{i}$. We argue by contradiction. Consider $\left|x_{n}\right| \rightarrow \infty$ and let $l=\lim _{\sup _{n \rightarrow \infty}} u_{\epsilon}\left(x_{n}, t\right)$. By extracting a subsequence we can assume that $l=\lim _{n \rightarrow \infty} u_{\epsilon}\left(x_{n}, t\right)$. Since $W$ is a $C^{2}$ function it holds that $\lim _{n \rightarrow \infty} W^{\prime}(u)\left(x_{n}, t\right)=W^{\prime}(l) \neq 0$ which contradicts our hypothesis. Therefore there must exist an $i$ such that $u\left(x_{n}, t\right) \rightarrow a_{i}$ for some $i$ and $v\left(x_{n}, t\right) \rightarrow W\left(a_{i}\right)$, which proves (14) and concludes the proof of the Lemma.

Notice that since this lemma is true for every $\bar{t}$ it holds that $|W(u)(x, t)| \leq \max \left\{W\left(\xi_{\epsilon}\right)\right.$, $\left.W\left(a_{i}\right)\right\}$ for every $(x, t) \in \mathbb{R}^{2} \times \mathbb{R}_{+}$.

Remark 2.1 If $-1 \leq \xi_{\epsilon}(x) \leq 1$ for every $x$, it is possible to show, when $u_{\epsilon}$ is single valued, that $-1 \leq u_{\epsilon}(x, t) \leq 1$. This fact follows by comparison principle, since $1,-1$ are solutions to (11). Since this proof cannot be generalized for vector valued solutions $u_{\epsilon}$, we chose to present here Lemma 2.1, that can be easily generalized to that setting, by adding the following assumption: there is a constant $C$ such that $W^{\prime \prime}(u)$ is positive definitive when $|u|>C$.

Lemma 2.2 Let $C$ be the constant given by Theorem 2.1. Then there is a constant $D \geq C$ and a positive potential $\tilde{W}$ that satisfies:

- $\tilde{W}(u) \equiv W(u)$ for $|u| \leq D$.

- $\left|\tilde{W}^{\prime \prime}(u)\right| \leq M$ for some constant $M$.

- $\tilde{W}^{\prime}(u)=0$ if and only if $W^{\prime}(u)=0$. That is $W$ and $\tilde{W}$ have the same critical points. 
Proof Let $D>C, \delta>0$ and

$$
\tilde{W}(u)=\eta(u) W(u)+(1-\eta(u)) f(u)
$$

where $\eta$ is a smooth function such that $\eta(u) \equiv 1$ for $|u| \leq D, \eta(u) \equiv 0$ for $|u| \geq 2 D$, $\eta^{\prime}(u) \leq 0$ for $D<u<D+\delta, \eta^{\prime}(u) \geq 0$ for $-D-\delta<u<-D$ and

$$
f(u)=\left\{\begin{array}{cc}
m_{1} u+d_{1} & \text { if } u \geq 0 \\
-m_{2} u+d_{2} & \text { if } u \leq 0
\end{array}\right.
$$

By definition $\tilde{W}=W(u)$ for $|u| \leq D$ and

$$
\tilde{W}^{\prime \prime}(u)=\eta^{\prime \prime}(u)(W(u)-f(u))+2 \eta^{\prime}(u) W^{\prime}(u)+\eta(u) W^{\prime \prime}(u)+(1-\eta(u)) f^{\prime \prime}(u)
$$

For $u \leq D$ this is equal $W^{\prime \prime}(u)$, if $D \leq|u| \leq D+\delta$ all these quantities are bounded (because $W, \eta$ and $f$ are $C^{2}$ for $\left.D \leq|u| \leq 2 \bar{D}\right)$ and $\tilde{W}^{\prime \prime}(u)=0$ otherwise. We are only left to show that we can choose $m_{i}, d_{i}$ and $D$ such that $\tilde{W}^{\prime}(u) \neq 0$ for $u \neq a_{i}$, where $\left\{a_{i}\right\}$ are the critical points of $W$. First we need $D$ such that $\left|a_{i}\right| \leq D$ for every $i$, then

$$
\tilde{W}^{\prime}(u)=W^{\prime}(u) \text { for }|u| \leq D,
$$

hence

$$
\tilde{W}^{\prime}(u)=0 \quad \text { if and only if } W^{\prime}(u)=0 .
$$

Since $W$ is proper, we can also choose $D$ so that $W$ is monotone for $D \leq|u| \leq D+\delta$ (increasing for $u>0$ and decreasing if $u<0$ ). Then we have

$$
\tilde{W}^{\prime}(u)=\eta^{\prime}(u)\left(W(u)-m_{1} u-d_{1}\right)+\eta(u)\left(W^{\prime}(u)-m_{1}\right)+m_{1} \quad \text { if } u \geq D
$$

and

$$
\tilde{W}^{\prime}(u)=\eta^{\prime}(u)\left(W(u)+m_{2} u-d_{2}\right)+\eta(u)\left(W^{\prime}(u)+m_{2}\right)-m_{2} \quad \text { if } u \leq-D
$$

Since for $|u| \geq D+\delta$ we have $\tilde{W}^{\prime}(u)=m_{i}$, we need $m_{i} \neq 0$. For $D$ large enough we have that $\max _{u \in[D, D+\delta]} W^{\prime}(u)>0$, hence choosing $m_{1}=\max _{u \in[D, D+\delta]} W^{\prime}(u)$ we have that

$$
\eta(u)\left(W^{\prime}(u)-m_{1}\right)+m_{1}>0 .
$$

Since $\eta^{\prime}(u) \leq 0$ for $D \leq u \leq D+\delta$ for $d_{1}$ large enough we have that

$$
\eta^{\prime}(u)\left(W(u)-m_{1} u-d_{1}\right) \geq 0
$$

concluding that

$$
\tilde{W}^{\prime}(u)>0 \text { for } D \leq u \leq D+\delta .
$$

By the definition of $\eta$ we have that $\tilde{W}^{\prime}(u)=m_{1}>0$ for $u \geq D+\delta$. Hence, $\tilde{W}^{\prime}(u)>0$ for $u \geq D$. Similarly, $m_{2}, d_{2}$ can be chosen such that $\tilde{W}^{\prime}(u)<0$ for $u \leq-D$, therefore $\tilde{W}^{\prime}(u) \neq 0$ for $|u| \geq D$, which concludes the proof.

We conclude this section by presenting some well known results for the function $u_{*}$, solution (5)-(6). Notice first that multiplying (5) by $\left(u_{*}\right)_{x}(x)$ we obtain

$$
-\left(\left(u_{*}\right)_{x}^{2}\right)_{x}+\left(\frac{W\left(u_{*}\right)}{2}\right)_{x}=0 .
$$


Integrating follows

$$
\left(u_{*}\right)_{x}^{2}=\frac{W\left(u_{*}\right)}{2}+A
$$

for $A=\left(u_{*}\right)_{x}^{2}(0)-\frac{W(0)}{2}$. Choosing the $\left(u_{*}\right)_{x}(0)$ such that the constant $A=0$ we have that $u_{*}(x)$ is defined implicitly for every $x \in \mathbb{R}$ by

$$
x=\int_{0}^{u_{*}(x)} \frac{d y}{\sqrt{W(y)}}, \quad-1<y<1
$$

From (15) we conclude that when $u_{*}$ approaches \pm 1 it holds that $x$ approaches $\pm \infty$. Since $\left(u_{*}\right)_{x}(x)=\sqrt{W\left(u_{*}\right)}>0$ it must hold that $\lim _{x \rightarrow \pm \infty} u_{*}(x)= \pm 1$ respectively.

We also have that the following lemma:

Lemma 2.3 Let $u_{*}$ be the solution to (5)-(6). Then for every $n \in \mathbb{N}$ there are constants $c_{n}$, $\gamma_{+}$and $\gamma_{-}$such that

$$
\begin{gathered}
\left|\frac{d^{(n)}\left(u_{*}(x)+1\right)}{d x^{n}}\right| \leq c_{n} e^{\gamma_{-} x} \quad \text { for all } x \leq 0 \\
\left|\frac{d^{(n)}\left(u_{*}(x)-1\right)}{d x^{n}}\right| \leq c_{n} e^{-\gamma_{+} x} \quad \text { for all } x \geq 0 ;
\end{gathered}
$$

where $\gamma_{ \pm}>0$ are defined by

$$
\gamma_{ \pm}^{2}=\frac{1}{4} W^{\prime \prime}( \pm 1)
$$

The proof of Lemma 2.3 is standard.

\section{Existence and estimates}

In this section we are going to prove existence for equation (11)-(12). Although an alternative proof can be found in [1], we will include our new method, since it provides bounds that will be useful in the following sections.

We are going to work with the following Banach spaces:

$$
C_{\left[\bar{t}_{1}, \bar{t}_{2}\right]}=\left\{u: \mathbb{R}^{2} \times\left[\bar{t}_{1}, \bar{t}_{2}\right] \rightarrow \mathbb{R}: u \text { is a uniformly bounded continuous function }\right\}
$$

with the standard $C^{0}$ norm, and its subspace given by:

$B_{\left[\bar{t}_{1}, \bar{t}_{2}\right]}=\left\{u: \mathbb{R}^{2} \times\left[\bar{t}_{1}, \bar{t}_{2}\right] \rightarrow \mathbb{R}: u \in C_{\left[\bar{t}_{1}, \bar{t}_{2}\right]}, W^{\prime}(u)(x, t) \rightarrow 0\right.$ as $|x| \rightarrow \infty$ for all $\left.t \in\left[\bar{t}_{1}, \bar{t}_{2}\right]\right\}$.

It is easy to see that $B_{\left[\bar{t}_{1}, \bar{t}_{2}\right]}$ is a Banach space with $C^{0}$ norm.

Consider some $\tau \geq 0$ and define $F_{\epsilon}^{\tau}: C_{[\tau, \bar{t}]} \rightarrow C_{[\tau, \bar{t}]}$ by

$$
F_{\epsilon}^{\tau}(u)(x, t)=\int_{\tau}^{t} \int_{\mathbb{R}^{2}} \mathcal{H}(x-y, t-s) \frac{-W^{\prime}(u)(y, s)}{2 \epsilon^{2}} d y d s+\int_{\mathbb{R}^{2}} \mathcal{H}(x-y, t-\tau) \xi_{\epsilon}^{\tau}(y) d y
$$

where $\mathcal{H}(x, t)$ is the heat kernel in $\mathbb{R}^{2}$. 
Notice that a solution $u_{\epsilon}$ of (11) such that $u_{\epsilon}(x, \tau)=\xi_{\epsilon}^{\tau}(x)$ is a fixed point of $F_{\epsilon}^{\tau}(\cdot)$, and conversely (in particular in this case the functional $F_{\epsilon}^{\tau}(\cdot)$ will depend on $u_{\epsilon}$ since $\xi_{\epsilon}^{\tau}$ does). In order to prove the following theorem (Theorem 3.1) we will find a fixed points of $F_{\epsilon}^{\tau}$ and find estimates for these fixed points. More precisely we show:

Theorem 3.1 Let $W: \mathbb{R} \rightarrow \mathbb{R}$ be a $\mathcal{C}^{2}$ function that satisfies

- $W(u) \rightarrow \infty$ as $|u| \rightarrow \infty$,

- there is a $\delta>0$ such that $\left|W^{\prime}(v)\right| \geq \delta$ as $|v| \rightarrow \infty$

- all the critical points of $W$ are contained in a compact set.

Then given a uniformly bounded initial conditions $\xi_{\epsilon}(x) \equiv \xi_{\epsilon}^{0}(x)$ such that $W^{\prime}\left(\xi_{\epsilon}\right)(x) \rightarrow$ 0 as $|x| \rightarrow \infty$, there is a uniformly bounded continuous function $u_{\epsilon}$ that satisfies (11)-(12) for $(x, t) \in \mathbb{R}^{2} \times(0, \infty)$.

Moreover, suppose that $\left\{\xi_{\epsilon}\right\}$ are uniformly bounded as $\epsilon \rightarrow 0$. Then, for every $\bar{t}>0$, $0<\epsilon \leq 1, K>0$ and sequence of continuous function $v_{\epsilon}(x, t)$ satisfying $\left|v_{\epsilon}(x, t)\right| \leq K$ and $\sup _{(x, t) \in \mathbb{R}^{2} \times[0, \bar{t}]}\left|u_{\epsilon}-v_{\epsilon}\right|(x, t) \mid \not \rightarrow 0$, there is a constant $C=C(K)$, independent of $\epsilon, \xi_{\epsilon}$ and $v_{\epsilon}$, that satisfies:

$$
\sup _{(x, t) \in \mathbb{R}^{2} \times[0, \bar{t}]}\left|u_{\epsilon}-v_{\epsilon}\right|(x, t) \leq C \sup _{(x, t) \in \mathbb{R}^{2} \times[0, \bar{t}]}\left|F_{\epsilon}^{0}\left(v_{\epsilon}\right)-v_{\epsilon}\right|(x, t),
$$

Moreover, $u_{\epsilon}$ satisfies for every $t>0$ that $W^{\prime}\left(u_{\epsilon}\right)(x, t) \rightarrow 0$ as $|x| \rightarrow \infty$.

Remark 3.1 Inequality (19) in particular implies that every sequence of functions $v_{\epsilon}$ such that $\left|v_{\epsilon}\right| \leq K$ and $\sup _{(x, t) \in \mathbb{R}^{2} \times[0, \bar{t}]}\left|F_{\epsilon}^{0}\left(v_{\epsilon}\right)-v_{\epsilon}\right| \rightarrow 0$, necessarily satisfies $\sup _{(x, t) \in \mathbb{R}^{2} \times[0, \bar{t}]} \mid u_{\epsilon}-$ $v_{\epsilon} \mid(x, t) \rightarrow 0$

To prove this theorem we will use the following lemmas.

Lemma 3.1 Suppose that there is a constant $M$ such that $\left|W^{\prime \prime}\right| \leq M$. Let $\xi_{\epsilon}^{\tau}$ be uniformly bounded. Then $F_{\epsilon}^{\tau}(\cdot): C_{[\tau, \bar{t}]} \rightarrow C_{[\tau, \bar{t}]}$ is well defined for each $\epsilon>0$. If additionally $\alpha \in(0,1)$ and $\bar{t}=\tau+\frac{2 \alpha \epsilon^{2}}{M}$, then $F_{\epsilon}^{\tau}$ is a contraction mapping with constant $\alpha$ in $\mathbb{R}^{2} \times[\tau, \bar{t}]$.

Proof The continuity of $W^{\prime}$ implies that if $\sup _{(x, t) \in \mathbb{R}^{2} \times[\tau, \bar{t}]}|u(x, t)| \leq C$, then there is a some constant $C_{1}$ such that $\sup _{(x, t) \in \mathbb{R}^{2} \times[\tau, \bar{t}]}\left|W^{\prime}(u)(x, t)\right| \leq C_{1}$. Therefore

$$
\begin{aligned}
\left|F_{\epsilon}^{\tau}(u)\right|(x, t) & \leq \frac{C_{1}}{\epsilon^{2}} \int_{\tau}^{\bar{t}} \int_{\mathbb{R}^{2}} \mathcal{H}(x-y, t-\tau-s) d y d s+\sup _{x \in \mathbb{R}^{2}}\left|\xi_{\epsilon}^{\tau}(x)\right| \int_{\mathbb{R}^{2}} \mathcal{H}(x-y, t-\tau) d y \\
& \leq \frac{C_{1}(\bar{t}-\tau)}{2 \epsilon^{2}}+\sup _{x \in \mathbb{R}^{2}}\left|\xi_{\epsilon}^{\tau}\right|(x)=\bar{C}<\infty,
\end{aligned}
$$

for all $(x, t)$. Hence $F_{\epsilon}^{\tau}$ is well defined for each $\epsilon>0$.

Since $\left|W^{\prime \prime}\right| \leq M$ we have that

$$
\left|W^{\prime}(u)-W^{\prime}(v)\right| \leq M|u-v| .
$$

Then for every $x \in \mathbb{R}^{2}$ and $t \in[\tau, \bar{t}]$ it holds that

$$
\begin{aligned}
\left|F_{\epsilon}^{\tau}(u)-F_{\epsilon}^{\tau}(v)\right|(x, t) & =\left|\int_{\tau}^{t} \int_{\mathbb{R}^{2}} \mathcal{H}(x-y, t-s-\tau) \frac{-W^{\prime}(u)(y, s)+W^{\prime}(v)(y, s)}{2 \epsilon^{2}} d y d s\right| \\
& \leq \frac{M(\bar{t}-\tau)}{2 \epsilon^{2}} \sup _{(x, t) \in \mathbb{R}^{2} \times[\tau, \bar{t}]}|u-v|(x, t) .
\end{aligned}
$$


Choosing $\bar{t}=\tau+\frac{2 \alpha \epsilon^{2}}{M}$ the previous inequality implies

$$
\sup _{(x, t) \in \mathbb{R}^{2} \times[\tau, \bar{t}]}\left|F_{\epsilon}^{\tau}(u)-F_{\epsilon}^{\tau}(v)\right|(x, t) \leq \alpha \sup _{(x, t) \in \mathbb{R}^{2} \times[\tau, \bar{t}]}|u-v|(x, t)
$$

Therefore $F_{\epsilon}^{\tau}: \mathbb{R}^{2} \times[\tau, \bar{t}] \rightarrow \mathbb{R}^{2} \times[\tau, \bar{t}]$ is a contraction with constant $\alpha$.

Lemma 3.2 Let $\tau_{1}<\tau_{2}<t$. Then for every continuous function $v_{\epsilon}$ one has

$$
\sup _{(x, t) \in \mathbb{R}^{2} \times\left[\tau_{2}, t\right]}\left|F_{\epsilon}^{\tau_{1}}\left(v_{\epsilon}\right)-F_{\epsilon}^{\tau_{2}}\left(v_{\epsilon}\right)\right| \leq \sup _{x \in \mathbb{R}^{2}}\left|\xi_{\epsilon}^{\tau_{2}}(x)-F_{\epsilon}^{\tau_{1}}\left(v_{\epsilon}\right)\left(x, \tau_{2}\right)\right| .
$$

Proof Let $V_{\epsilon}(x, t)=F_{\epsilon}^{\tau_{1}}\left(v_{\epsilon}\right)(x, t)-F_{\epsilon}^{\tau_{2}}\left(v_{\epsilon}\right)(x, t)$. By definition we have for every $t>\tau_{2}$ that

$$
P V_{\epsilon}=\frac{W^{\prime}\left(v_{\epsilon}\right)}{2 \epsilon^{2}}-\frac{W^{\prime}\left(v_{\epsilon}\right)}{2 \epsilon^{2}}=0
$$

and

$$
V_{\epsilon}\left(x, \tau_{2}\right)=\xi_{\epsilon}^{\tau_{2}}(x)-F_{\epsilon}^{\tau_{1}}\left(v_{\epsilon}\right)\left(x, \tau_{2}\right) .
$$

Duhamel's formula implies that for every $t>\tau_{2}$

$$
V_{\epsilon}(x, t)=\int_{\mathbb{R}^{2}} \mathcal{H}\left(x-y, t-\tau_{2}\right)\left(\xi_{\epsilon}^{\tau_{2}}(x)-F_{\epsilon}^{\tau_{1}}\left(v_{\epsilon}\right)\right)\left(y, \tau_{2}\right) d y .
$$

Hence, for every $x \in \mathbb{R}^{2}$ and $t \geq \tau_{2}$

$$
\left|V_{\epsilon}\right|(x, t) \leq \sup _{y \in \mathbb{R}^{2}}\left|\xi_{\epsilon}^{\tau_{2}}(y)-F_{\epsilon}^{\tau_{1}}\left(v_{\epsilon}\right)\right|\left(y, \tau_{2}\right),
$$

which finishes the proof of (20).

\section{Lemma 3.3 If}

- $W(u) \rightarrow \infty$ as $|u| \rightarrow \infty$,

- there is a $\delta>0$ such that $W^{\prime}(u)>\delta$ when $|u| \rightarrow \infty$,

- all the critical points of $W$ are contained in a compact set and

- $\xi_{\epsilon}^{\tau}$ is such that $W^{\prime}\left(\xi_{\epsilon}^{\tau}\right)(x) \rightarrow 0$ as $|x| \rightarrow \infty$,

then

$$
F_{\epsilon}^{\tau}\left(C_{[\tau, \bar{t}]} \bigcap B_{[\tau, \bar{t}]}\right) \subset C_{[\tau, \bar{t}]} \bigcap B_{[\tau, \bar{t}]} .
$$

Proof Consider $u \in C_{[\tau, \bar{t}]} \cap B_{[\tau, \bar{t}]}$. Let us define

$$
I_{1}(x, t)=\int_{\tau}^{t} \int_{\mathbb{R}^{2}} \mathcal{H}(x-y, t-s) \frac{-W^{\prime}(u)(y, s)}{2 \epsilon^{2}} d y d s
$$

and

$$
I_{2}(x, t)=\int_{\mathbb{R}^{2}} \mathcal{H}(x-y, t-\tau) \xi_{\epsilon}^{\tau}(y) d y .
$$

Since $F_{\epsilon}^{\tau}(u)(x, t)=I_{1}(x, t)+I_{2}(x, t)$, we are interested in analyzing the behavior of $I_{1}(x, t)$ and $I_{2}(x, t)$ as $x \rightarrow \infty$. 
Changing variables, we have

$$
I_{1}=\int_{0}^{t} \int_{\mathbb{R}^{2}} \mathcal{H}(y, s) \frac{-W^{\prime}(u)(x-y, t-s)}{2 \epsilon^{2}} d y d s .
$$

By hypothesis $W^{\prime}(u)(x, t) \rightarrow 0$ as $|x| \rightarrow \infty$, for every $y, t$ and $s$. We also know that $W$ is a $C^{2}$ function and $u$ is uniformly bounded, so $\left|W^{\prime}(u)(x-y, t-\tau-s)\right|$ is uniformly bounded in $\mathbb{R}^{2} \times[\tau, \bar{t}]$ and

$$
\left|\mathcal{H}(y, s) \frac{-W^{\prime}(u)(x-y, t-\tau-s)}{2 \epsilon^{2}}\right| \leq C(\epsilon) \mathcal{H}(y, s) \in L^{1}\left(\mathbb{R}^{2} \times[\tau, \bar{t}]\right) .
$$

By dominated convergence it follows that

$$
I_{1}(x, t) \int_{\tau}^{t} \int_{\mathbb{R}^{2}} \mathcal{H}(y, s) \frac{-W^{\prime}(u)(x-y, t-\tau-s)}{2 \epsilon^{2}} d y d s \rightarrow 0 \text { as }|x| \rightarrow \infty .
$$

This implies that $\lim _{|x| \rightarrow \infty} F_{\epsilon}^{\tau}(x, t)$ depends on the behavior of $I_{2}(x, t)$ as $x$ approaches infinity. As before we have that.

$$
I_{2}(x, t)=\int_{\mathbb{R}^{2}} \mathcal{H}(y, t-\tau) \xi_{\epsilon}^{\tau}(x-y) d y
$$

We are going to prove the result of the lemma by contradiction. Suppose that $\lim _{x \rightarrow \infty} W^{\prime}\left(F_{\epsilon}^{\tau}(u)\right)(x, t)$ is not 0 or it does not exist. Then there is a sequence $\left\{x_{n}\right\}_{n \in \mathbb{N}}$ such that $x_{n} \rightarrow \infty$ as $n \rightarrow \infty$ and either $\lim _{n \rightarrow \infty} W^{\prime}\left(F_{\epsilon}^{\tau}(u)\right)\left(x_{n}, t\right)=c \neq 0$ or it is infinite. By hypothesis

$$
W^{\prime}\left(\xi_{\epsilon}^{\tau}\right)\left(x_{n}\right) \rightarrow 0 \text { as } n \rightarrow \infty .
$$

Given the assumptions over $W$ it must hold that $\xi_{\epsilon}^{\tau}\left(x_{n}\right)$ is uniformly bounded in $\mathbb{R}^{2}$. This implies that there exists a convergent subsequence of $\left\{\xi_{\epsilon}^{\tau}\left(x_{n}\right)\right\}_{n \in \mathbb{N}}$, which we relabel $\left\{\xi\left(x_{n}\right)\right\}_{n \in \mathbb{N}}$. Let $\lim _{n \rightarrow \infty} \xi_{\epsilon}^{\tau}\left(x_{n}\right)=l<\infty$; then by using (22) and dominated convergence, we have that

$$
\lim _{n \rightarrow \infty} \int_{\mathbb{R}^{2}} \mathcal{H}\left(x_{n}-y, t-\tau\right) \xi_{\epsilon}^{\tau}(y) d y=l .
$$

Combining (21) and (24) we conclude that

$$
\lim _{n \rightarrow \infty} F_{\epsilon}^{\tau}(u)\left(x_{n}, t\right)=l .
$$

Using this equality and the continuity of $W^{\prime}$ we have

$$
\lim _{n \rightarrow \infty} W^{\prime}\left(F_{\epsilon}^{\tau}(u)\right)\left(x_{n}, t\right)=W^{\prime}(l)
$$

but by (23) one has

$$
W^{\prime}(l)=\lim _{n \rightarrow \infty} W^{\prime}\left(\xi\left(x_{n}\right)\right)=0
$$

so

$$
\lim _{n \rightarrow \infty} W^{\prime}\left(F_{\epsilon}^{\tau}(u)\right)\left(x_{n}, t\right)=0
$$

which contradicts the definition of $\left\{x_{n}\right\}_{n \in \mathbb{N}}$. Hence $\lim _{x \rightarrow \infty} W^{\prime}\left(F_{\epsilon}^{\tau}(u)\right)(x, t)=0$, which finishes the proof of Lemma 3.3. 
Lemma 3.4 If the function $W \in C^{2}$ satisfies:

- there is a constant $M$ such that $\left|W^{\prime \prime}\right| \leq M$,

- $W(u) \rightarrow \infty$ as $|u| \rightarrow \infty$,

- there is a $\delta>0$ such that $W^{\prime}(u)>\delta$ when $|u| \rightarrow \infty$,

- $\quad$ all the critical points of $W$ are contained in a compact set and

- $\xi_{\epsilon}^{\tau}$ is such that $W^{\prime}\left(\xi_{\epsilon}^{\tau}\right)(x) \rightarrow 0$ as $|x| \rightarrow \infty$,

then the function $F_{\epsilon}^{\tau}: C_{[\tau, \bar{t}]} \cap B_{[\tau, \bar{t}]} \rightarrow C_{[\tau, \bar{t}]} \cap B_{[\tau, \bar{t}]}$ is well defined for any given $\tau, \bar{t} \geq 0$. If additionally $\alpha \in(0,1)$ and $\bar{t}=\tau+\frac{2 \alpha \epsilon^{2}}{M}$, then $F_{\epsilon}^{\tau}$ is a contraction mapping with constant $\alpha$ in $\mathbb{R}^{2} \times[\tau, \bar{t}]$.

Proof This follows directly by combining Lemma 3.1 and Lemma 3.3.

Proof of Theorem 3.1: First we will assume that $\left|W^{\prime \prime}\right| \leq M$. In this case we will prove this Theorem in the following steps:

- Prove existence for small time intervals by using the contraction mapping $F_{\epsilon}^{\tau}$ defined by (18). The length of these time intervals depends on $\epsilon$ and $\alpha$, the contraction constant of $F_{\epsilon}^{\tau}$.

- Extend the solution to all times, by proving that the solutions and its derivatives match in the intersection of the chosen time intervals.

- Find estimates for each time interval.

- Use these local estimates to find a global estimate independent of $\epsilon$.

Step 1 Fix $\bar{t}>0$ and let

$$
\tau_{i}=i \frac{2 \alpha \epsilon^{2}}{M}
$$

with $i=0, \ldots, I_{\alpha}$, where the constant $\alpha, I_{\alpha} \in \mathbb{N}$ satisfy $\frac{\bar{t} M}{2 \alpha \epsilon^{2}} \leq I_{\alpha} \leq 2 \frac{\bar{t} M}{2 \alpha \epsilon^{2}}$. By the definition of $\tau_{i}$ we have that $\bar{t}_{I_{\alpha}} \geq \bar{t}$. We will redefine $\tau_{I_{\alpha}+1}=\bar{t}$.

Consider $v_{\epsilon}(x, t) \in C_{[0, \bar{t}]}$. Then we also have $v_{\epsilon}(x, t) \in C_{\left[\tau_{i}, \tau_{i+1}\right]}$. Let $F_{\epsilon, i}=F_{\epsilon}^{\tau_{i}}$. And we will denote by $F_{\epsilon, i}^{n}$ the composition of $F_{\epsilon, i}$ with itself $n$ times.

By Lemma 3.1 we have

$$
\sup _{\mathbb{R}^{2} \times\left[\tau_{i}, \tau_{i+1}\right]}\left|F_{\epsilon, i}^{n+1}\left(v_{\epsilon}\right)(x, t)-F_{\epsilon, i}^{n}\left(v_{\epsilon}\right)(x, t)\right| \leq \alpha^{n} \sup _{\mathbb{R}^{2} \times\left[\tau_{i}, \tau_{i+1}\right]}\left|F_{\epsilon, i}\left(v_{\epsilon}\right)(x, t)-v_{\epsilon}(x, t)\right| .
$$

where the supremum is taken over $\mathbb{R}^{2} \times\left[\tau_{i}, \tau_{i+1}\right]$. Now, by the usual arguments we conclude that $\left\{F_{\epsilon, i}^{n}\left(v_{\epsilon}\right)\right\}$ is a Cauchy sequence, which implies that there is a function $u_{\epsilon}^{i} \in C_{\left[\tau_{i}, \tau_{i+1}\right]}$ such that $F_{\epsilon, i}^{n}\left(v_{\epsilon}(x, t)\right) \rightarrow u_{\epsilon}^{i}(x, t)$ as $n \rightarrow \infty$. Also $F_{\epsilon, i}^{n+1}\left(v_{\epsilon}(x, t)\right)=F_{\epsilon, i}\left(F_{\epsilon, i}^{n}\left(v_{\epsilon}(x, t)\right)\right)$, so taking the limit as $n \rightarrow \infty$ we see that $u_{\epsilon}^{i}(x, t)$ satisfies

$$
F_{\epsilon, i}\left(u_{\epsilon}^{i}\right)(x, t)=u_{\epsilon}^{i}(x, t) .
$$

Since the heat kernel is smooth, we have that for any finite $\tau_{i}, \tau_{i+1}$, its convolution with a continuous function is well defined and smooth. It follows that for any continuous function $u$, the function $F_{\epsilon, i}(u)$ is in $C^{\infty}\left(\mathbb{R}^{2} \times\left[\tau_{i}, \tau_{i+1}\right]\right)$. In particular, by using (27) we have that $u_{\epsilon}^{i}(x, t)$ is $C^{\infty}\left(\mathbb{R}^{2} \times\left[\tau_{i}, \tau_{i+1}\right]\right)$. From (27) and Duhamel's formula we can conclude that $u_{\epsilon}^{i}$ satisfies

$$
\left(u_{\epsilon}^{i}\right)_{t}-\Delta u_{\epsilon}^{i}+\frac{1}{2 \epsilon^{2}} W^{\prime}\left(u_{\epsilon}^{i}\right)=0
$$




$$
u_{\epsilon}^{i}\left(x, \tau_{i}\right)=\xi_{\epsilon}^{\tau_{i}}(x)
$$

for $(x, t) \in \mathbb{R}^{2} \times\left(\tau_{i}, \tau_{i+1}\right)$.

Step 2 Now define recursively $\xi_{\epsilon}^{\tau_{i}}(x)$ :

$$
\begin{gathered}
\xi_{\epsilon}^{\tau_{0}}(x)=\xi_{\epsilon}(x) \\
\xi_{\epsilon}^{\tau_{i}}(x)=u_{\epsilon}^{i-1}\left(x, \tau_{i}\right) .
\end{gathered}
$$

Now we define $u_{\epsilon}(x, t)$ by

$$
u_{\epsilon}(x, t)=u_{\epsilon}^{i}(x, t) \quad \text { for } t \in\left[\tau_{i}, \tau_{i+1}\right] .
$$

It is clear that $u_{\epsilon}$ is a solution to (11) for $t \in\left(\tau_{i}, \tau_{i+1}\right)$. Moreover, by writing

$$
\begin{aligned}
u_{\epsilon}^{i+1}(x, t)= & \int_{\bar{t}_{i}}^{t} \int_{\mathbb{R}^{2}} \mathcal{H}\left(x-y, t-\bar{t}_{i}-s\right) \frac{-W^{\prime}\left(u_{\epsilon}^{i+1}\right)(y, s)}{2 \epsilon^{2}} d y d s \\
& +\int_{\mathbb{R}^{2}} \mathcal{H}\left(x-y, t-\bar{t}_{i}\right) u_{\epsilon}^{i}\left(y, \tau_{i+1}\right) d y,
\end{aligned}
$$

and taking derivatives it is clear that the space derivatives of $u_{\epsilon}$ are continuous functions of time. Using the continuity of the space derivatives and Eq. (11) for $u_{\epsilon}^{i+1}$ and $u_{\epsilon}^{i}$ we conclude that the time derivatives of $u_{\epsilon}$ are continuous in time as well. This fact easily implies that $u_{\epsilon}$ is a solution to (11) for all times. Moreover, by the definition of $u_{\epsilon}^{0}$, we have that $u_{\epsilon}$ also satisfies (12).

Step 3 Now we need to prove formula (19). Notice that using inequality (26) one has

$$
\sup _{\mathbb{R}^{2} \times\left[\tau_{i}, \tau_{i+1}\right]}\left|F_{\epsilon, i}^{n}\left(v_{\epsilon}\right)-v_{\epsilon}\right|(x, t) \leq \frac{1}{1-\alpha} \sup _{\mathbb{R}^{2} \times\left[\tau_{i}, \tau_{i+1}\right]}\left|F_{\epsilon, i}\left(v_{\epsilon}\right)-v_{\epsilon}\right|(x, t) .
$$

Taking the limit as $n \rightarrow \infty$

$$
\sup _{\mathbb{R}^{2} \times\left[\tau_{i}, \tau_{i+1}\right]}\left|u_{\epsilon}-v_{\epsilon}\right|(x, t) \leq \frac{1}{1-\alpha} \sup _{\mathbb{R}^{2} \times\left[\tau_{i}, \tau_{i+1}\right]}\left|F_{\epsilon, i}\left(v_{\epsilon}\right)-v_{\epsilon}\right|(x, t) .
$$

Using Lemma 3.2, this implies

$$
\begin{aligned}
\sup _{\mathbb{R}^{2} \times\left[\tau_{i}, \tau_{i+1}\right]}\left|u_{\epsilon}-v_{\epsilon}\right|(x, t) & \leq \frac{1}{1-\alpha}\left(\sup _{\mathbb{R}^{2} \times\left[\tau_{i}, \tau_{i+1}\right]}\left|F_{\epsilon, i}\left(v_{\epsilon}\right)-F_{\epsilon, 0}\left(v_{\epsilon}\right)\right|(x, t)\right. \\
& \left.\quad+\sup _{\mathbb{R}^{2} \times\left[\tau_{i}, \tau_{i+1}\right]}\left|F_{\epsilon, 0}\left(v_{\epsilon}\right)-v_{\epsilon}\right|(x, t)\right) \\
& \leq \frac{1}{1-\alpha}\left(\sup _{\mathbb{R}^{2}}\left|u_{\epsilon}-F_{\epsilon, 0}\left(v_{\epsilon}\right)\right|\left(x, \tau_{i}\right)+\sup _{\mathbb{R}^{2} \times\left[\tau_{i}, \tau_{i+1}\right]}\left|F_{\epsilon, 0}\left(v_{\epsilon}\right)-v_{\epsilon}\right|(x, t)\right) \\
& \leq \frac{1}{1-\alpha}\left(\sup _{\mathbb{R}^{2}}\left|u_{\epsilon}-v_{\epsilon}\right|\left(x, \tau_{i}\right)+2 \sup _{\mathbb{R}^{2} \times\left[\tau_{i}, \tau_{i+1}\right]}\left|F_{\epsilon, 0}\left(v_{\epsilon}\right)-v_{\epsilon}\right|(x, t)\right) .
\end{aligned}
$$


Recursively, we conclude that for every $T<\infty, \epsilon>0$ there is a constant $C$ such that

$$
\sup _{\mathbb{R}^{2} \times[0, T]}\left|u_{\epsilon}-v_{\epsilon}\right|(x, t) \leq C(T, \epsilon) \sup _{\mathbb{R}^{2} \times[0, T]}\left|F_{\epsilon, 0}\left(v_{\epsilon}\right)-v_{\epsilon}\right|(x, t) .
$$

A straightforward computations shows that the functions $u_{\epsilon}\left(\epsilon x, \epsilon^{2} t\right)$ satisfy (11), but

$$
u_{\epsilon}(\epsilon x, 0)=\xi_{\epsilon}(\epsilon x)
$$

That is, $u_{\epsilon}\left(\epsilon x, \epsilon^{2} t\right)$ is a fixed point of

$$
G_{\epsilon}(v)(x, t)=\int_{0}^{t} \int_{\mathbb{R}^{2}} \mathcal{H}(x-y, t-s) \frac{-W^{\prime}(v)(y, s)}{2} d y d s+\int_{\mathbb{R}^{2}} \mathcal{H}(x-y, t-\tau) \xi_{\epsilon}(\epsilon y) d y .
$$

Therefore (19) is equivalent to every $T>0$ and every continuous $v$, such that $\sup _{\mathbb{R}^{2} \times[0, T]} \mid u_{\epsilon}-$ $v_{\epsilon} \mid \geq \delta$ and $|v| \leq K$, satisfies

$$
\sup _{\mathbb{R}^{2} \times[0, T]}\left|u_{\epsilon}\left(\epsilon x, \epsilon^{2} t\right)-v(x, t)\right| \leq C \sup _{\mathbb{R}^{2} \times[0, T]}\left|G_{\epsilon}(v)-v\right|(x, t) .
$$

To simplify the notation we will denote in the remainder of the proof

$$
u_{\epsilon}^{\epsilon}(x, t)=u_{\epsilon}\left(\epsilon x, \epsilon^{2} t\right) .
$$

If (36) does not hold there are sequences of functions $u_{j}, v_{j}$ and a sequence of times $T_{j} \rightarrow T \in[0, \infty]$ such that $\left|u_{j}-v_{j}\right|(x, t) \geq \delta$ and

$$
\sup _{\mathbb{R}^{2} \times\left[0, T_{j}\right]}\left|u_{j}-v_{j}\right|(x, t) \geq j \sup _{\mathbb{R}^{2} \times\left[0, T_{j}\right]}\left|G_{j}\left(v_{j}\right)-v_{j}\right|(x, t),
$$

where $u_{j}(x, t)=u_{\epsilon_{j}}^{\epsilon_{j}}(x, t)$ and $G_{j}=G_{\epsilon_{j}}$.

Recall that $u_{j}$ and $v_{j}$ are uniformly bounded. Then, Eq. (37) implies that

$$
\sup _{\mathbb{R}^{2} \times\left[0, T_{j}\right]}\left|G_{j}\left(v_{j}\right)-v_{j}\right|(x, t) \rightarrow 0 \text { as } j \rightarrow \infty .
$$

We will show that (38) implies $\sup _{\mathbb{R}^{2} \times\left[0, T_{j}\right]}\left|u_{j}-v_{j}\right|(x, t) \rightarrow 0$, contradicting the assumption $\left|u_{j}-v_{j}\right|(x, t) \nrightarrow 0$.

Let $\mathcal{T}=\left\{\left(t_{j}\right)_{j \in \mathbb{N}}: t_{j} \leq T_{j}\right.$ and $\left.\sup _{\mathbb{R}^{2} \times\left[0, t_{j}\right]}\left|u_{j}-v_{j}\right|(x, t) \rightarrow 0\right\} \subset\left\{\left(t_{j}\right)_{j \in \mathbb{N}}: t_{j} \leq T_{j}\right\}$. We will show that the set $\mathcal{T}$ is non-empty, open and closed in $\left\{\left(t_{j}\right): t_{j} \leq T_{j}\right\}$ and then conclude that is the whole set.

Equation (35) implies that $\mathcal{T}$ is not empty (by choosing $t_{j}=T$, where $T$ is some constant time). Let us show now that $\mathcal{T}$ is closed.

Consider a sequence $\left(t_{j}^{n}\right)_{j} \in \mathcal{T}$ such that $t_{j}^{n} \rightarrow t_{j}$ as $n \rightarrow \infty$ and let us prove that $\left(t_{j}\right)_{j \in \mathbb{N}} \in \mathcal{T}$. Notice that

$$
\sup _{\mathbb{R}^{2} \times\left[0, t_{j}\right]}\left|u_{j}-v_{j}\right|(x, t) \leq \max \left\{\sup _{\mathbb{R}^{2} \times\left[0, t_{j}^{n}\right]}\left|u_{j}-v_{j}\right|(x, t), \sup _{\mathbb{R}^{2} \times\left[t_{j}^{n}, t_{j}\right]}\left|u_{j}-v_{j}\right|(x, t)\right\} .
$$


Since $\left(t_{j}^{n}\right) \in \mathcal{T}$ we only need to find bounds over $\sup _{\mathbb{R}^{2} \times\left[t_{j}^{n}, t_{j}\right]}\left|u_{j}-v_{j}\right|(x, t)$. Since for $n$ large enough we have that $t_{j}-t_{j}^{n} \leq \frac{2 \alpha}{M}$, from (34) we have that

$$
\begin{aligned}
& \sup _{\mathbb{R}^{2} \times\left[t_{j}^{n}, t_{j}\right]}\left|u_{j}-v_{j}\right|(x, t) \\
& \leq \frac{1}{1-\alpha}\left(\sup _{\mathbb{R}^{2}}\left|u_{1}-v_{j}\right|\left(x, t_{j}^{n}\right)+2 \sup _{\mathbb{R}^{2} \times\left[t_{j}^{n}, t_{j}\right]}\left|G_{j}\left(v_{j}\right)-v_{j}\right|(x, t)\right) \\
& \leq \frac{1}{1-\alpha}\left(\sup _{\mathbb{R}^{2} \times\left[0, t_{j}^{n}\right]}\left|u_{1}-v_{j}\right|(x, t)+2 \sup _{\mathbb{R}^{2} \times\left[0, T_{j}\right]}\left|G_{j}\left(v_{j}\right)-v_{j}\right|(x, t)\right) .
\end{aligned}
$$

This implies

$$
\begin{aligned}
& \sup _{\mathbb{R}^{2} \times\left[0, t_{j}\right]}\left|u_{j}-v_{j}\right|(x, t) \\
& \quad \leq \frac{1}{1-\alpha}\left(\sup _{\mathbb{R}^{2} \times\left[0, t_{j}^{n}\right]}\left|u_{1}-v_{j}\right|(x, t)+2 \sup _{\mathbb{R}^{2} \times\left[0, T_{j}\right]}\left|G_{j}\left(v_{j}\right)-v_{j}\right|(x, t)\right) .
\end{aligned}
$$

By a standard diagonal argument, combined with equation (38) we have $\sup _{\mathbb{R}^{2} \times\left[0, t_{j}\right]} \mid u_{j}-$ $v_{j} \mid(x, t) \rightarrow 0$ as $j \rightarrow \infty$, and hence $\left(t_{j}\right)_{j \in \mathbb{N}} \in \mathcal{T}$.

In order to show that $\mathcal{T}$ is open consider $\left(\tilde{T}_{j}\right)_{j} \in \mathcal{T}$ and define $S_{j}=\tilde{T}_{j}+\min \left\{\frac{2 \alpha}{M}, T_{j}-\tilde{T}_{j}\right\}$ $\leq T_{j}$. Equation (34) implies for every $j$ holds that

$$
\begin{aligned}
& \sup _{\mathbb{R}^{2} \times\left[0, S_{j}\right]}\left|u_{j}-v_{j}\right|(x, t) \\
& \leq \frac{1}{1-\alpha}\left(\sup _{\mathbb{R}^{2}}\left|u_{1}-v_{j}\right|\left(x, \tilde{T}_{j}\right)+2 \sup _{\mathbb{R}^{2} \times\left[0, T_{j}\right]}\left|G_{j}\left(v_{j}\right)-v_{j}\right|(x, t)\right) .
\end{aligned}
$$

Then

$$
\begin{aligned}
& \sup _{\mathbb{R}^{2} \times\left[0, S_{j}\right]}\left|u_{j}-v_{j}\right|(x, t) \\
& \quad \leq \frac{1}{1-\alpha}\left(\sup _{\mathbb{R}^{2} \times\left[0, \tilde{T}_{j}\right]}\left|u_{1}-v_{j}\right|(x, t)+2 \sup _{\mathbb{R}^{2} \times\left[0, T_{j}\right]}\left|G_{j}\left(v_{j}\right)-v_{j}\right|(x, t)\right) .
\end{aligned}
$$

Since $\tilde{T}_{j} \in \mathcal{T}$, using Eq. (38), we have that $\sup _{\mathbb{R}^{2} \times\left[0, S_{j}\right]}\left|u_{j}-v_{j}\right|(x, t) \rightarrow 0$ as $j \rightarrow \infty$, hence $\left(S_{j}\right)_{j \in \mathbb{N}} \in \mathcal{T}$. We conclude that $\left\{\left(t_{j}\right)_{j}: t_{j} \leq S_{j}\right\} \subset \mathcal{T}$. Since $\tilde{T}_{j}<S_{j}$ for every $\tilde{T}_{j} \neq T_{j}$, necessarily $\left\{\left(t_{j}\right)_{j}: t_{j} \leq S_{j}\right\}$ contains an open neighborhood of $\left(\tilde{T}_{j}\right)_{j}$, hence $\mathcal{T}$ is also open.

Finally, since $\mathcal{T}$ is open closed and non-empty, we have that $\mathcal{T}=\left\{\left(t_{j}\right)_{j}: t_{j} \leq T_{j}\right\}$. In particular $\sup _{\mathbb{R}^{2} \times\left[0, T_{j}\right]}\left|u_{j}-v_{j}\right|(x, t) \rightarrow 0$ as $j \rightarrow \infty$, which contradicts the assumption. Therefore, (36) must hold, finishing the proof of Theorem 3.1 when $\left|W^{\prime \prime}\right|$ is unifomly bounded.

For the general case (that is when $W^{\prime \prime}$ is not necessarily bounded) we can repeat the proof for $\tilde{W}$ constructed in Lemma 2.2. Lemma 2.1 implies that the fixed point that we find for $\tilde{W}$ is also a solution to (11)-(12) and equation (19) holds for any $\left|v_{\epsilon}\right| \leq D$. 
Remark 3.2 Notice that when $v_{\epsilon}$ is regular enough we can write

$$
v_{\epsilon}(x, t)=\int_{0}^{t} \int_{\mathbb{R}^{2}} P v_{\epsilon}(y, s) d y d s+\int_{\mathbb{R}^{2}} \mathcal{H}(x-y, t) v_{\epsilon}(y, 0) d y .
$$

Hence, from inequality (19) and the definition of $F$ we derive, when $v_{\epsilon}$ is a family of continuous functions such that $\left|v_{\epsilon}\right| \leq K$ and $\sup _{\mathbb{R}^{2} \times[0, \bar{t}]}\left|v_{\epsilon}-u_{\epsilon}\right| \not \rightarrow 0$ as $\epsilon \rightarrow 0$, that there is a constant $C$ independent of $\epsilon$ such that:

$$
\begin{aligned}
& \sup _{\mathbb{R}^{2} \times[0, \bar{t}]}\left|u_{\epsilon}-v_{\epsilon}\right|(x, t) \\
& \leq C\left(\sup _{(x, t) \in \mathbb{R}^{2} \times[0, \bar{t}]} \int_{0}^{t} \int_{\mathbb{R}^{2}} \mathcal{H}(x-y, t-s)\left|\frac{-W^{\prime}\left(v_{\epsilon}\right)}{2 \epsilon^{2}}-P v_{\epsilon}\right|(y, s) d y d s\right. \\
&\left.\quad+\sup _{(x, t) \in \mathbb{R}^{2} \times[0, \bar{t}]} \int_{\mathbb{R}^{2}} \mathcal{H}(x-y, t)\left|\xi_{\epsilon}(y)-v_{\epsilon}(y, 0)\right| d y\right) .
\end{aligned}
$$

\section{Proof of Theorems 1.1 and 1.2}

Let $\Gamma(\lambda, t) \subset \mathbb{R}^{2}$ parametrized by the space parameter $\lambda \in[0, \bar{\lambda}]$, be a compact curve flowing by mean curvature, that is, for any parametrization holds

$$
\frac{\partial \Gamma}{\partial t} \cdot v=k_{\Gamma}(\lambda, t)
$$

where $k_{\Gamma}$ is the curvature of $\Gamma$ and $v$ its normal vector.

Let $d(x, t)$ be the signed distance from $x$ to $\Gamma(\lambda, t)$. Recall that where $d$ is smooth

$$
|\nabla d|=1 \text {. }
$$

Since $\Gamma$ is a smooth curve, there is a constant $\tau>0$ such that for $|d| \leq \tau, d(x, t)$ is a smooth function. Using these facts and standard computations we have the following lemma (see [1] for example):

Lemma 4.1 Let $\Gamma(\lambda, t)$ be flowing by mean curvature and $d(x, t)$ the signed distance to $\Gamma(\lambda, t)$ then it holds

$$
d_{t}-\Delta d=\frac{k^{2}(\lambda, t) d}{1+k(\lambda, t) d}
$$

where $k(\lambda, t)$ is the curvature of $\Gamma$ at time $t$.

Since we have freedom in choosing the initial condition we are going to define the following function $\psi_{\epsilon}$ (which satisfies the conditions of Theorem 1.1) that will be the initial condition in the rest of this paper: Fix $\chi \in \mathcal{C}_{0}^{\infty}((-1,1)),|\chi| \leq 1$ and $\chi(x)=1$ for $x \in\left[-\frac{1}{2}, \frac{1}{2}\right]$; and set

$$
\psi_{\epsilon}(x)=\chi(d(x, 0)) u_{*}\left(\frac{d(x, 0)}{\epsilon}\right)+(1-\chi(d(x, 0))) \frac{d(x, 0)}{|d(x, 0)|} .
$$

Now we can prove Theorem 1.1. 
Proof of Theorem 1.1: The strategy will be the following: first we construct a uniformly bounded function $v_{\epsilon}$ that satisfies (8)-(9) and such that the function $\left|P v_{\epsilon}(y, s)+\frac{W^{\prime}\left(v_{\epsilon}\right)(y, s)}{2 \epsilon^{2}}\right|$ is also uniformly bounded (independently of $\epsilon$ ); moreover, it converges to 0 as $\epsilon \rightarrow 0$ and is supported in a neighborhood of $\Gamma$. We conclude using inequality (39), with $v_{\epsilon}$ as a test function, and Dominated Convergence Theorem.

Since $\Gamma(\lambda, t)$ is smooth for all $0<t \leq \bar{T}$ there is $\bar{\tau}$ such that for $|d| \leq \bar{\tau}$ the function $d(x, t)$ is a smooth for every $t \leq \bar{T}$. Consider $-1 \leq \chi_{\bar{\tau}} \leq 1$ be a $C^{\infty}$ function with support in $[-\bar{\tau}, \bar{\tau}]$ and $\chi_{\bar{\tau}}(x)=1$ for $x \in\left[-\frac{\bar{\tau}}{2}, \frac{\bar{\tau}}{2}\right]$.

We can define

$$
v_{\epsilon}(x, t)=\chi_{\bar{\tau}}(d(x, t)) u_{*}\left(\frac{d(x, t)}{\epsilon}\right)+\left(1-\chi_{\bar{\tau}}\right) \frac{d(x, t)}{|d(x, t)|} .
$$

where $u_{*}$ is defined by (5)-(6). The function $v_{\epsilon}$ satisfies

$$
\begin{aligned}
P v_{\epsilon}(x, t)+\frac{W^{\prime}\left(v_{\epsilon}\right)(x, t)}{2 \epsilon^{2}}= & \left(\chi_{\bar{\tau}}^{\prime} d_{t}-\chi_{\bar{\tau}}^{\prime} \Delta d-\chi_{\bar{\tau}}^{\prime \prime}|\nabla d|^{2}\right)\left(u_{*}-\frac{d}{|d|}\right)-2 \chi_{\bar{\tau}}^{\prime}|\nabla d|^{2} \frac{u_{*}^{\prime}}{\epsilon} \\
& +\frac{\chi_{\bar{\tau}} u_{*}^{\prime}}{\epsilon}\left(d_{t}-\Delta d\right)-\frac{\chi_{\bar{\tau}}|\nabla d|^{2} W^{\prime}\left(u_{*}\right)}{2 \epsilon^{2}}+\frac{W^{\prime}\left(v_{\epsilon}\right)}{2 \epsilon^{2}}
\end{aligned}
$$

Notice that for $d \leq-\tau_{\bar{T}}$ and $d \geq \tau_{\bar{T}}$ we have that $\tilde{u}_{\epsilon}= \pm 1$ respectively, hence

$$
P v_{\epsilon}(x, t)+\frac{W^{\prime}\left(v_{\epsilon}\right)(x, t)}{2 \epsilon^{2}}=0 .
$$

That is $P v_{\epsilon}(x, t)+\frac{W^{\prime}\left(v_{\epsilon}\right)(x, t)}{2 \epsilon^{2}}$ is supported in a neighborhood of $\Gamma$.

Using Lemma 4.1, (16), (17) and (44) we prove that for every $x$ such that $-\bar{\tau}_{\bar{T}} \leq d(x, t) \leq$ $\bar{\tau}_{\bar{T}}, P v_{\epsilon}(x, t)+\frac{W^{\prime}\left(v_{\epsilon}\right)(x, t)}{2 \epsilon^{2}}$ converges pointwise to 0 and it is uniformly bounded independently of $\epsilon$. Namely, we have for $\gamma=\min \left\{\gamma_{+}, \gamma_{-}\right\}$

- If $x$ satisfies $\frac{\bar{\tau}}{2} \leq|d(x, t)| \leq \bar{\tau}$, then

$$
\left|P v_{\epsilon}(x, t)+\frac{W^{\prime}\left(v_{\epsilon}\right)(x, t)}{2 \epsilon^{2}}\right| \leq C \frac{e^{-\gamma / \bar{\tau}} \frac{\epsilon^{2 \epsilon}}{\epsilon^{2}}}{\text { if } \frac{\bar{\tau}}{2}} \leq|d(x, t)| \leq \bar{\tau} .
$$

- For $x$ such that $|d(x, t)| \leq \frac{\bar{\tau}}{2}$ we have, by definition of $\chi_{\tau}$, that

$$
\left|P v_{\epsilon}(x, t)+\frac{W^{\prime}\left(v_{\epsilon}\right)(x, t)}{2 \epsilon^{2}}\right|=\frac{u_{*}^{\prime}}{\epsilon}\left(d_{t}-\Delta d\right) \leq C \frac{d e^{-\gamma \frac{d}{\epsilon}}}{\epsilon} .
$$

It follows from equations (45) and (46) that $\left|P v_{\epsilon}(x, t)+\frac{W^{\prime}\left(v_{\epsilon}\right)(x, t)}{2 \epsilon^{2}}\right|$ converges pointwise to 0 for every fixed $(x, t)$. Notice that when $d(x, t)=0$ it holds for every $t$ and $\epsilon>0$ that $\left|P v_{\epsilon}(x, t)+\frac{W^{\prime}\left(v_{\epsilon}\right)(x, t)}{2 \epsilon^{2}}\right|=0$.

The uniform boundedness also follows from Eqs. (45) and (46) by noticing that for every $\epsilon>0$ the quantities $\frac{e^{-\gamma \frac{\bar{\tau}}{2 \epsilon}}}{\epsilon^{2}}$ and $\frac{d e^{-\gamma \frac{d}{\epsilon}}}{\epsilon}$ can be bounded by a constant that only depends on $\gamma$ and $\bar{\tau}$. We would like to point out that this bounds are possible away form the first singularity time. Similar estimates can be found in [1]. 
Inequality (39) implies that

$$
\begin{aligned}
\sup _{\mathbb{R}^{2} \times[0, \bar{T}]}\left|u_{\epsilon}-v_{\epsilon}\right|(x, t) \leq & C \sup _{\mathbb{R}^{2} \times[0, \bar{T}]} \int_{0}^{t} \int_{\mathbb{R}^{2}} \mathcal{H}(x-y, t-s)\left|\frac{-W^{\prime}\left(v_{\epsilon}\right)(y, s)}{2 \epsilon^{2}}-P v_{\epsilon}(y, s)\right| d y d s \\
& +C \sup _{(x, t) \in \mathbb{R}^{2} \times[0, \bar{T}]} \int_{\mathbb{R}^{2}} \mathcal{H}(x-y, t)\left|\psi_{\epsilon}(y)-v_{\epsilon}(y, 0)\right| d y .
\end{aligned}
$$

For simplicity

$$
\mathcal{S}_{\epsilon}(x, t)=\int_{0}^{t} \int_{\mathbb{R}^{2}} \mathcal{H}(x-y, t-s)\left|\frac{W^{\prime}\left(v_{\epsilon}\right)(y, s)}{2 \epsilon^{2}}+P v_{\epsilon}(y, s)\right| d y d s .
$$

Consider any $0<t<\bar{T}$ and $\delta>0$. Then, using (45) and (46), we have a constant $C$, independent of $\epsilon$, such that

$$
\begin{aligned}
\mathcal{S}_{\epsilon}(x, t) \leq & \int_{0}^{t-\delta} \int_{B_{R}} \mathcal{H}(x-y, t-s)\left|\frac{W^{\prime}\left(v_{\epsilon}\right)(y, s)}{2 \epsilon^{2}}+P v_{\epsilon}(y, s)\right| d y d s \\
& +C \int_{t-\delta}^{t} \int_{\mathbb{R}^{2}} \mathcal{H}(x-y, t-s)+C \int_{0}^{t-\delta} \int_{\mathbb{R}^{2} \backslash B_{R}} \mathcal{H}(x-y, t-s) d y d s .
\end{aligned}
$$

For $R$ large enough (independent of $x, t$ ) the last integral is smaller than $\delta$ (notice that if $\Gamma$ is compact it is just identically 0$)$. Also by the definition of $\mathcal{H}$ we have for every $x, y \in \mathbb{R}^{2}$ and $s \leq t-\delta$, that $\mathcal{H}(x-y, t-s) \leq \frac{C}{\delta}$. We conclude that for every $x$ holds

$$
\begin{aligned}
\mathcal{S}_{\epsilon}(x, t) & \leq \frac{C}{\delta} \int_{0}^{t-\delta} \int_{B_{R}}\left|\frac{-W^{\prime}\left(v_{\epsilon}\right)(y, s)}{2 \epsilon^{2}}-P v_{\epsilon}(y, s)\right| d y d s+2 C \delta \\
& \leq \frac{C}{\delta} \int_{0}^{T} \int_{B_{R}}\left|\frac{-W^{\prime}\left(v_{\epsilon}\right)(y, s)}{2 \epsilon^{2}}-P v_{\epsilon}(y, s)\right| d y d s+2 C \delta .
\end{aligned}
$$

Using Dominated Convergence Theorem and equations (45)-(46) we can see that

$$
\lim _{\epsilon \rightarrow 0} \sup _{\mathbb{R}^{2} \times[0, \bar{T}]} \mathcal{S}_{\epsilon}(x, t) \leq 2 C \delta
$$

Since this holds for every $\delta>0$ we have

$$
\lim _{\epsilon \rightarrow 0} \sup _{\mathbb{R}^{2} \times[0, \bar{T}]} \mathcal{S}_{\epsilon}(x, t)=0 .
$$

Let us prove now that $\lim _{\epsilon \rightarrow 0} \sup \left|v_{\epsilon}(y, 0)-\psi_{\epsilon}(y)\right|=0$. Without loss of generality we can assume that the width $\left(\tau_{\bar{t}}\right)$ of the tubular neigborhood of $\Gamma$ such that $d$ is smooth is less than 1 , that is $1 \geq \tau_{\bar{t}}$. Therefore we have that

$$
v_{\epsilon}(x, 0)-\psi_{\epsilon}(y)= \begin{cases}0 & \text { if }|d| \leq \frac{\bar{\tau}}{2} \text { or }|d| \geq 1 \\ \left(u_{*}-\frac{d}{|d|}\right)\left(\chi-\chi_{\bar{\tau}}\right) & \text { otherwise. }\end{cases}
$$


Equations (16) and (17) imply that

$$
\left|v_{\epsilon}(y, 0)-\psi_{\epsilon}(y)\right| \leq C e^{-\frac{\gamma}{2 \epsilon}} .
$$

It follows from (39)

$$
\lim _{\epsilon \rightarrow 0} \sup _{\mathbb{R}^{2} \times[0, \bar{T}]}\left|u_{\epsilon}-v_{\epsilon}\right|(x, t) \leq C \lim _{\epsilon \rightarrow 0} \sup _{\mathbb{R}^{2} \times[0, \bar{T}]} \mathcal{S}_{\epsilon}(x, t)+\lim _{\epsilon \rightarrow 0} \bar{T} C e^{-\frac{\gamma}{2 \epsilon}}=0
$$

finishing the proof.

Now we proceed to show Theorem 1.2.

Proof of Theorem 1.2: We will use the following notation. Let $T$ be the maximal time of existence of $\Gamma$ and $x_{0}=\Gamma(\cdot, T)$. Let

$$
\begin{gathered}
r=\left|x-x_{0}\right|, \\
R_{T}(t)=\sqrt{2(T-t)} \\
a(x, t, \epsilon)=d(x, t)+\frac{\epsilon^{2}|\ln \epsilon|^{3+p}}{R_{T}(t)},
\end{gathered}
$$

$\eta$ compactly supported such that

$$
\eta(x, y)=\left\{\begin{array}{ll}
1 & \text { if }|x| \leq 1 \text { and }|y| \leq 1 \\
0 & \text { if }|x| \geq 2 \text { or }|y| \geq 2
\end{array} .\right.
$$

and

$$
\begin{aligned}
\mathcal{A}(r, t, \epsilon)= & \left(1-\eta\left(\frac{\left|x-x_{0}\right|}{\epsilon R_{T}(t)}, \frac{R_{T}^{2}(t)}{3 \epsilon^{2}|\ln \epsilon|^{3+p}}\right)\right) a(r, t, \epsilon) \\
& +\eta\left(\frac{\left|x-x_{0}\right|}{\epsilon R_{T}(t)}, \frac{R_{T}^{2}(t)}{3 \epsilon^{2}|\ln \epsilon|^{3+p}}\right)\left(-R_{T}(t)+\frac{\epsilon^{2}|\ln \epsilon|^{3+p}}{R_{T}(t)}\right) .
\end{aligned}
$$

Define

$$
v_{\epsilon}^{*}(r, t)=\left\{\begin{array}{cl}
\chi\left(\frac{\mathcal{A}(r, t, \epsilon)}{R(t)}\right) u_{*}\left(\frac{\mathcal{A}(r, t, \epsilon)}{\epsilon}\right)+\left(1-\chi\left(\frac{\mathcal{A}(r, t, \epsilon)}{R(t)}\right)\right) \frac{\mathcal{A}(r, t, \epsilon)}{|\mathcal{A}(r, t, \epsilon)|} & \text { for } t \leq 1 \\
1 & \text { for } t \geq 1
\end{array}\right.
$$

where $\chi$ is defined as before ( that is $-1 \leq \chi \leq 1$ is a $C^{\infty}$ function with support in $[-1,1]$ and $\chi(x)=1$ for $\left.x \in\left[-\frac{1}{2}, \frac{1}{2}\right]\right)$.

We will show that $u_{\epsilon}$, solution to (11)-(12) (where $\psi_{\epsilon}$ is defined by (42)), satisfies

$$
\lim _{\epsilon \rightarrow 0} \sup _{(x, t) \in \mathbb{R}^{2} \times[0, \infty)}\left|u_{\epsilon}(x, t)-v_{\epsilon}^{*}(x, t)\right|=0 .
$$

First we show that

$$
\sup _{\mathbb{R}^{2} \times[0, \infty)}\left|u_{\epsilon}-v_{\epsilon}^{*}\right|(x, t)=\sup _{\mathbb{R}^{2} \times[0, T]}\left|u_{\epsilon}-v_{\epsilon}^{*}\right|(x, t) .
$$

Let

$$
\begin{gathered}
u_{\epsilon}^{T}(x, t)=u_{\epsilon}(x, t+T) \\
\left(v_{\epsilon}^{*}\right)^{T}(x, t)=v_{\epsilon}^{*}(x, t+T) \equiv 1 .
\end{gathered}
$$


Since $P 1=W^{\prime}(1)=0$ and $v_{\epsilon}^{*}$ is continuous and bounded for every $\epsilon>0$, using inequality (39) we have for $x \in \mathbb{R}^{2}$ and $t \geq T$ that

$$
\left|u_{\epsilon}(x, t)-v_{\epsilon}^{*}(x, t)\right| \leq \sup _{y \in \mathbb{R}^{2}}\left|u_{\epsilon}(y, T)-v_{\epsilon}^{*}(y, T)\right| \leq \sup _{(y, t) \in \mathbb{R}^{2} \times[0, T]}\left|u_{\epsilon}(y, t)-v_{\epsilon}^{*}(y, t)\right|,
$$

proving (48).

To show that $\lim _{\epsilon \rightarrow 0} \sup _{(y, t) \in \mathbb{R}^{2} \times[0, T]}\left|u_{\epsilon}(y, t)-v_{\epsilon}^{*}(y, t)\right|=0$ we will use the result of Theorem 5.1 and argue by contradiction.

Suppose that

$$
\lim _{\epsilon \rightarrow 0} \sup _{(y, t) \in \mathbb{R}^{2} \times[0, T]}\left|u_{\epsilon}(y, t)-v_{\epsilon}^{*}(y, t)\right| \neq 0 .
$$

Then there must exist $\delta>0$ and sequences $\left\{\epsilon_{n}\right\} \subset[0, T],\left\{x_{n}\right\} \subset \mathbb{R}^{2},\left\{t_{n}\right\} \subset[0, T]$ such that $\epsilon_{n} \rightarrow 0$ as $n \rightarrow \infty$ and

$$
\left|u_{\epsilon_{n}}\left(x_{n}, t_{n}\right)-v_{\epsilon_{n}}^{*}\left(x_{n}, t_{n}\right)\right|>\delta .
$$

Since $t_{n} \in[0, T]$ for every $n$, there is a subsequence, that we relabel as $t_{n}$ such that $t_{n} \rightarrow \bar{t}$.

We divide the rest of the proof in two cases: $\bar{t}<T$ and $\bar{t}=T$.

- $\bar{t}<T$ Let $\bar{t}<\tilde{t}<T$. Then there is an $n_{0}$ such that for every $n>n_{0}$ it holds $t_{n}<\tilde{t}$. This implies for every $n>n_{0}$ that

$$
\begin{aligned}
\left|u_{\epsilon_{n}}\left(x_{n}, t_{n}\right)-v_{\epsilon_{n}}^{*}\left(x_{n}, t_{n}\right)\right| \leq & \sup _{(y, t) \in \mathbb{R}^{2} \times[0, \tilde{t}]}\left|u_{\epsilon_{n}}(y, t)-v_{\epsilon_{n}}^{*}(y, t)\right| \\
\leq & \sup _{(y, t) \in \mathbb{R}^{2} \times[0, \tilde{t}]}\left|u_{\epsilon_{n}}(y, t)-v_{\epsilon_{n}}(y, t)\right| \\
& +\sup _{(y, t) \in \mathbb{R}^{2} \times[0, \tilde{t}]}\left|v_{\epsilon_{n}}(y, t)-v_{\epsilon_{n}}^{*}(y, t)\right|
\end{aligned}
$$

where $v_{\epsilon_{n}}(y, t)$ in the function defined by (43). Definitions (43) and (47) imply that

$$
\sup _{(y, t) \in \mathbb{R}^{2} \times[0, \tilde{t}]}\left|v_{\epsilon_{n}}(y, t)-v_{\epsilon_{n}}^{*}(y, t)\right| \rightarrow 0 \quad \text { as } n \rightarrow \infty .
$$

Since $\bar{t}<T$, by Theorem 1.1 we have

$$
\sup _{(y, t) \in \mathbb{R}^{2} \times[0, \tilde{t}]}\left|u_{\epsilon_{n}}(y, t)-v_{\epsilon_{n}}(y, t)\right| \rightarrow 0 \quad \text { as } n \rightarrow \infty .
$$

Hence

$$
\left|u_{\epsilon_{n}}\left(x_{n}, t_{n}\right)-v_{\epsilon_{n}}^{*}\left(x_{n}, t_{n}\right)\right| \rightarrow 0 \quad \text { as } n \rightarrow \infty
$$

contradicting (49).

- $\bar{t}=T$ Consider a circle $C_{T}(t)$ centered at $x_{0}$ with radius $R_{T}(t)$. Let $u_{\epsilon}^{C}(x, t)$ and $v_{\epsilon}^{C}(x, t)$ be respectively the solution to (11) and the approximation to this solution given by Theorem 5.1. For every $n$ we have that

$$
\begin{aligned}
\left|u_{\epsilon_{n}}\left(x_{n}, t_{n}\right)-v_{\epsilon_{n}}^{*}\left(x_{n}, t_{n}\right)\right| \leq & \sup _{y \in \mathbb{R}^{2}}\left|u_{\epsilon_{n}}\left(y, t_{n}\right)-u_{\epsilon_{n}}^{C}\left(y, t_{n}\right)\right| \\
& +\sup _{y \in \mathbb{R}^{2}}\left|u_{\epsilon_{n}}^{C}(y, t)-v_{\epsilon_{n}}^{C}(y, t)\right| \\
& +\sup _{y \in \mathbb{R}^{2}}\left|v_{\epsilon_{n}}^{C}\left(y, t_{n}\right)-v_{\epsilon_{n}}^{*}\left(y, t_{n}\right)\right| .
\end{aligned}
$$


Theorem 5.1 implies that

$$
\sup _{y \in \mathbb{R}^{2}}\left|u_{\epsilon_{n}}^{C}(y, t)-v_{\epsilon_{n}}^{C}(y, t)\right| \rightarrow 0 \quad \text { as } n \rightarrow \infty .
$$

Using definitions (52) and (47) we have

$$
\sup _{y \in \mathbb{R}^{2}}\left|v_{\epsilon_{n}}^{C}\left(y, t_{n}\right)-v_{\epsilon_{n}}^{*}\left(y, t_{n}\right)\right| \rightarrow 0 \quad \text { as } n \rightarrow \infty .
$$

Let us show now that there are subsequences of $\left\{\epsilon_{n}\right\},\left\{t_{n}\right\}$ such that $\sup _{y \in \mathbb{R}^{2}}\left|u_{\epsilon_{n}}^{C}\left(y, t_{n}\right)-u_{\epsilon_{n}}\left(y, t_{n}\right)\right|$ converges to 0 as $n \rightarrow \infty$.

Let $w_{\epsilon, n}(x, t)$ for $t \geq t_{n}$ be the solution to (11) such that $w_{\epsilon, n}\left(x, t_{n}\right)=v_{\epsilon}^{*}\left(x, t_{n}\right)$. Respectively, we consider $w_{\epsilon, n}^{C}(x, t)$ for $t \geq t_{n}$ to be the solution to (11) such that $w_{\epsilon, n}^{C}\left(x, t_{n}\right)=v_{\epsilon}^{C}\left(x, t_{n}\right)$. Theorem 1.1 implies that for every fixed $n$ holds

$$
\begin{aligned}
& D_{m}\left(t_{n}\right) \equiv \sup _{x \in \mathbb{R}^{2}}\left|u_{\epsilon_{m}}\left(x, t_{n}\right)-v_{\epsilon_{m}}^{*}\left(x, t_{n}\right)\right| \rightarrow 0 \quad \text { as } m \rightarrow \infty, \\
& D_{m}^{C}\left(t_{n}\right) \equiv \sup _{x \in \mathbb{R}^{2}}\left|u_{\epsilon_{m}}^{C}\left(x, t_{n}\right)-v_{\epsilon_{m}}^{C}\left(x, t_{n}\right)\right| \rightarrow 0 \quad \text { as } m \rightarrow \infty .
\end{aligned}
$$

Therefore we can define

$$
m(n)=\inf \left\{m \in \mathbb{N}: m \geq n, D_{n}(j), C_{n}(j) \leq \frac{1}{n} \text { for every } j \geq m\right\} .
$$

Without loss of generality we can assume that $t_{n}$ are increasing (maybe by passing to a subsequence), hence we have $t_{m(n)} \geq t_{n}$. Then for the subsequences $\left\{\epsilon_{m(n)}\right\},\left\{t_{m(n)}\right\}$ holds

$$
\begin{aligned}
\sup _{x \in \mathbb{R}^{2}}\left|u_{\epsilon_{m(n)}}\left(x, t_{m(n)}\right)-u_{\epsilon_{m(n)}}^{C}\left(x, t_{m(n)}\right)\right| \leq & \sup _{x \in \mathbb{R}^{2}}\left|u_{\epsilon_{m(n)}}\left(x, t_{m(n)}\right)-w_{\epsilon_{m(n)}, n}\left(x, t_{m(n)}\right)\right| \\
& +\sup _{x \in \mathbb{R}^{2}}\left|w_{\epsilon_{m(n)}, n}\left(x, t_{m(n)}\right)-w_{\epsilon_{m(n)}, n}^{C}\left(x, t_{m(n)}\right)\right| \\
& +\sup _{x \in \mathbb{R}^{2}}\left|w_{\epsilon_{m(n)}, n}^{C}\left(x, t_{m(n)}\right)-u_{\epsilon_{m(n)}}^{C}\left(x, t_{m(n)}\right)\right| .
\end{aligned}
$$

Using inequality (39) we conclude

$$
\begin{aligned}
\sup _{x \in \mathbb{R}^{2}}\left|u_{\epsilon_{m(n)}}\left(x, t_{m(n)}\right)-u_{\epsilon_{m(n)}}^{C}\left(x, t_{m(n)}\right)\right| \leq & \sup _{x \in \mathbb{R}^{2}}\left|u_{\epsilon_{m(n)}}\left(x, t_{n}\right)-v_{\epsilon_{m(n)}}^{*}\left(x, t_{n}\right)\right| \\
& +\sup _{x \in \mathbb{R}^{2}}\left|v_{\epsilon_{m(n)}}^{*}\left(x, t_{n}\right)-v_{\epsilon_{m(n)}}^{C}\left(x, t_{n}\right)\right| \\
& +\sup _{x \in \mathbb{R}^{2}}\left|v_{\epsilon_{m(n)}}^{C}\left(x, t_{n}\right)-u_{\epsilon_{m(n)}}^{C}\left(x, t_{n}\right)\right| \\
\leq & \frac{2}{n}+\sup _{x \in \mathbb{R}^{2}}\left|v_{\epsilon_{m(n)}}^{*}\left(x, t_{n}\right)-v_{\epsilon_{m(n)}}^{C}\left(x, t_{n}\right)\right| .
\end{aligned}
$$

Using definitions (52) and (47) we have $\sup _{x \in \mathbb{R}^{2}}\left|u_{\epsilon_{m(n)}}\left(x, t_{m(n)}\right)-u_{\epsilon_{m(n)}}^{C}\left(x, t_{m(n)}\right)\right| \rightarrow 0$ as $n \rightarrow \infty$.

We conclude that there is a subsequence of $\left\{\epsilon_{n}\right\},\left\{t_{n}\right\}$ (defined as above) such that $\sup _{y \in \mathbb{R}^{2}}\left|u_{\epsilon_{n}}^{C}\left(y, t_{n}\right)-u_{\epsilon_{n}}\left(y, t_{n}\right)\right| \rightarrow 0$ as $n \rightarrow \infty$, contradicting (49) and finishing the proof of Theorem 1.2. 


\section{Appendix}

In this appendix we show Theorem 1.2 for a particular case. Namely, we consider a circle flowing under its curvature and prove:

Theorem 5.1 Let $R(t)$ be the radius at time t of a unit circle evolving under curve shortening flow, that is

$$
R(t)=\sqrt{2(1-t)} .
$$

Consider $\eta \in C_{0}^{\infty}(\mathbb{R} \times \mathbb{R}), 0 \leq \eta \leq 1$ and

$$
\eta(x, y)= \begin{cases}1 & \text { if }|x| \leq 1 \text { and }|y| \leq 1 \\ 0 & \text { if }|x| \geq 2 \text { or }|y| \geq 2\end{cases}
$$

Define

$$
a(t, \epsilon)=R(t)-\frac{\epsilon^{2}|\ln \epsilon|^{3+p}}{R(t)}
$$

and

$\mathcal{A}(r, t, \epsilon)=\left(1-\eta\left(\frac{r}{\epsilon R(t)}, \frac{R^{2}(t)}{3 \epsilon^{2}|\ln \epsilon|^{3+p}}\right)\right)(r-a(t, \epsilon))-\eta\left(\frac{r}{\epsilon R(t)}, \frac{R^{2}(t)}{3 \epsilon^{2}|\ln \epsilon|^{3+p}}\right) a(t, \epsilon)$.

Let $r=|x|$ and consider the function

$$
v_{\epsilon}^{*}(r, t)= \begin{cases}\chi\left(\frac{\mathcal{A}}{R(t)}\right) u_{*}\left(\frac{\mathcal{A}(r, t, \epsilon)}{\epsilon}\right)+\left(1-\chi\left(\frac{\mathcal{A}}{R(t)}\right)\right) \frac{\mathcal{A}(r, t, \epsilon)}{|\mathcal{A}(r, t, \epsilon)|} & \text { for } t \leq 1 \\ 1 & \text { for } t \geq 1,\end{cases}
$$

where $\chi: \mathbb{R} \rightarrow \mathbb{R}$ is a positive compactly supported function that satisfies

$$
\chi(x)= \begin{cases}1 & \text { if }|x| \leq \frac{1}{4} \\ 0 & \text { if }|x| \geq \frac{1}{2}\end{cases}
$$

Then $u_{\epsilon}$ satisfies

$$
\lim _{\epsilon \rightarrow 0} \sup _{(x, t) \in \mathbb{R}^{2} \times[0, \infty)}\left|u_{\epsilon}(x, t)-v_{\epsilon}^{*}(x, t)\right|=0 .
$$

Proof of Theorem 5.1: In order to prove this theorem we use the same strategy used to prove Theorem 1.1. That is, we construct a function $v_{\epsilon}^{*}$ with the required behavior, that also satisfies appropriate bounds for $P v_{\epsilon}^{*}+\frac{W^{\prime}\left(v_{\epsilon}\right)}{2 \epsilon^{2}}$. Then we use the inequality provided by Theorem 3.1 to conclude the result.

As in the proof of Theorem 1.2 we have that

$$
\sup _{\mathbb{R}^{2} \times[0, \infty)}\left|u_{\epsilon}-v_{\epsilon}^{*}\right|(x, t) \leq C \sup _{\mathbb{R}^{2} \times[0,1]}\left|u_{\epsilon}-v_{\epsilon}^{*}\right|(x, t) .
$$

Using Theorem 3.1 we have that:

$$
\begin{aligned}
\sup _{t \in[0, \bar{t}], x \in \mathbb{R}^{2}}\left|u_{\epsilon}-v_{\epsilon}\right|(x, t) \leq C & {\left[\sup _{\mathbb{R}^{2} \times[0, \bar{t}]} \int_{0}^{t} \int_{\mathbb{R}^{2}} \mathcal{H}(x-y, t-s)\left|\frac{-W^{\prime}\left(v_{\epsilon}^{*}\right)}{2 \epsilon^{2}}-P v_{\epsilon}^{*}\right|(y, s) d y d s\right.} \\
& \left.+\sup _{y \in \mathbb{R}^{2}}\left|\psi_{\epsilon}(y)-v_{\epsilon}^{*}(y, 0)\right| d y\right] .
\end{aligned}
$$


By definition of $v_{\epsilon}^{*}$ we have that

$$
\left|\psi_{\epsilon}(x)-v_{\epsilon}^{*}(x, 0)\right|=\left|v_{\epsilon}(r, 0)-v_{\epsilon}\left(r-\epsilon^{2}|\ln \epsilon|^{3+p}, 0\right)\right| .
$$

Hence

$$
\lim _{\epsilon \rightarrow 0} \sup _{x \in \mathbb{R}^{2}}\left|\psi_{\epsilon}(x)-v_{\epsilon}^{*}(x, 0)\right|=0 .
$$

Hence, we only need to show that

$$
\int_{0}^{t} \int_{\mathbb{R}^{2}} \mathcal{H}(x-y, t-s)\left|\frac{\left.-W^{\prime}\left(v_{\epsilon}^{*}\right)\right)}{2 \epsilon^{2}}-P v_{\epsilon}^{*}\right|(y, s) d y d s
$$

converges uniformly to 0 as $\epsilon \rightarrow 0$.

Let us start by computing $\frac{-W^{\prime}\left(v_{\epsilon}^{*}(y, s)\right)}{2 \epsilon^{2}}-P v_{\epsilon}^{*}(y, s)$. Notice first that when $\mathcal{A}=0$ we have that $\left(1-\chi\left(\frac{\mathcal{A}}{R(t)}\right)\right) \frac{\mathcal{A}}{|\mathcal{A}|}=0$. Hence $v_{\epsilon}^{*}$ is smooth and well defined, moreover:

$$
\begin{aligned}
P v_{\epsilon}^{*}+\frac{W^{\prime}\left(v_{\epsilon}^{*}\right)}{2 \epsilon^{2}}= & \left(u_{*}\left(\frac{\mathcal{A}}{\epsilon}\right)-\frac{\mathcal{A}}{|\mathcal{A}|}\right)\left[\frac{\chi^{\prime}}{R(t)}\left(\mathcal{A}_{t}+\frac{\mathcal{A}}{R^{2}(t)}-\frac{\mathcal{A}_{r}}{r}-\mathcal{A}_{r r}\right)\right. \\
& \left.-\chi^{\prime \prime} \frac{\mathcal{A}_{r}^{2}}{R^{2}(t)}\right]+\frac{\chi u_{*}^{\prime}}{\epsilon}\left[\mathcal{A}_{t}-\frac{\mathcal{A}_{r}}{r}-\mathcal{A}_{r r}\right] \\
& -2 \frac{\chi^{\prime} u_{*}^{\prime}}{R(t) \epsilon} \mathcal{A}_{r}^{2}-\frac{\chi W^{\prime}\left(u_{*}\right)}{2 \epsilon^{2}} \mathcal{A}_{r}^{2}+\frac{W^{\prime}\left(v_{\epsilon}^{*}\right)}{2 \epsilon^{2}} .
\end{aligned}
$$

Where

$$
\begin{aligned}
\mathcal{A}_{t} & =\left(\frac{1}{R(t)}+\frac{\epsilon^{2}|\ln \epsilon|^{3+p}}{R^{3}(t)}\right)+r\left(\frac{\eta_{x} r}{\epsilon R^{3}(t)}+\frac{\eta_{y}}{\epsilon^{2}|\ln \epsilon|^{3+p}}\right), \\
\mathcal{A}_{r} & =(1-\eta)-\frac{r}{\epsilon R(t)} \eta_{x}, \\
\mathcal{A}_{r r} & =-\frac{2}{\epsilon R(t)} \eta_{x}-\frac{r}{\epsilon^{2} R^{2}(t)} \eta_{x x} .
\end{aligned}
$$

We used the following abbreviations:

$$
u_{*}^{(n)}=\frac{d^{n} u_{*}}{d x^{n}}\left(\frac{\mathcal{A}}{\epsilon}\right), \quad \chi^{(n)}=\frac{d^{n} \chi}{d x^{n}}\left(\frac{\mathcal{A}}{R(t)}\right) .
$$

For simplicity, we denote

$$
f_{\epsilon}(x, t)=\left|P v_{\epsilon}^{*}+\frac{W^{\prime}\left(v_{\epsilon}^{*}\right)}{2 \epsilon^{2}}\right|(x, t) .
$$

and

$$
F_{\epsilon}(x, t)=\int_{0}^{t} \int_{\mathbb{R}^{2}} \mathcal{H}(x-y, t-s) f_{\epsilon}(y, s) d y d s .
$$

In order to prove Theorem 5.1 we will show that the positive function

$$
F_{\epsilon}(x, t)=\int_{0}^{t} \int_{\mathbb{R}^{2}} \mathcal{H}(x-y, t-s) f_{\epsilon}(y, s) d y d s
$$

converges uniformly to 0 as $\epsilon \rightarrow 0$. 
As in the proof of Theorem 1.1 we start by finding pointwise estimates for $f_{\epsilon}$.

Before finding bounds for $f_{\epsilon}$, we would like to point out that for every $t$ such that $R(t) \leq$ $2 \epsilon^{2}|\ln \epsilon|^{3+p}$ holds that $\mathcal{A} \geq 1$, for $\epsilon$ small enough. This implies that $v^{*}(x, t)=1$ and $f(x, t)=0$. Hence, when bounding $f_{\epsilon}$, it is enough to consider $t \leq t_{*}$, where $t_{*}$ is the time such that

$$
R\left(t_{*}\right)=2 \epsilon^{2}|\ln \epsilon|^{3+p} .
$$

Estimates (16) and (17) imply that

$$
\left|u_{*}-\frac{\mathcal{A}}{|\mathcal{A}|}\right|,\left|u^{\prime}\right|,\left|u^{\prime}\right|,\left|u^{\prime \prime}\right| \leq C e^{-\gamma \frac{|\mathcal{A}|}{\epsilon}} .
$$

Notice that if $t$ is such that $R^{2}(t) \geq 2 \epsilon^{2}|\ln \epsilon|^{3+p}$ or $r \geq \epsilon R(t)$ we have that $\mathcal{A}_{r}=1$. Then, as in the proof of Theorem 1.1

$$
\left|\frac{\chi W^{\prime}\left(u_{*}\right)}{2 \epsilon^{2}} \mathcal{A}_{r}^{2}+\frac{W^{\prime}\left(v_{\epsilon}^{*}\right)}{2 \epsilon^{2}}\right| \leq C \frac{\left|u_{*}-\frac{\mathcal{A}}{|\mathcal{A}|}\right|}{\epsilon^{2}} .
$$

Then, (59) and (60) yield for $t$ such that $R^{2}(t) \geq 6 \epsilon^{2}|\ln \epsilon|^{3+p}$ or $r \geq \epsilon R(t)$, when $|\mathcal{A}| \geq-K \epsilon \ln \epsilon>0$ (that is when $r$ is "far enough" from the evolving circle of radius $R(t)$ ) that for the constant $\gamma$ defined above

$$
\left|u_{*}-\frac{\mathcal{A}}{|\mathcal{A}|}\right|,\left|u^{\prime}\right|,\left|u^{\prime}\right|\left|u^{\prime \prime}\right|,\left|\frac{\chi W^{\prime}\left(u_{*}\right)}{2 \epsilon^{2}} \mathcal{A}_{r}^{2}+\frac{W^{\prime}\left(v_{\epsilon}^{*}\right)}{2 \epsilon^{2}}\right| \leq C \epsilon^{\gamma K} .
$$

We want to use this bound for every $r, t$ such that $|\mathcal{A}| \geq-\epsilon \ln \epsilon$ and either $t$ satisfies $R^{2}(t) \geq 6 \epsilon^{2}|\ln \epsilon|^{3+p}$ or $r \geq \epsilon R(t)$.

Notice that when $R^{2}(t) \geq 6 \epsilon^{2}|\ln \epsilon|^{3+p}$ and $\frac{\mathcal{A}}{R(t)} \geq-\frac{1}{2}$, by the definition of $\mathcal{A}$, holds $r \geq \sqrt{2} \epsilon|\ln \epsilon|^{\frac{3+p}{2}}$. Then (54) and (61) imply

$$
\left|f_{\epsilon}(r, t)\right| \leq \frac{C \epsilon^{\gamma K}}{\epsilon^{2}} .
$$

We can trivially extend this bound when $\frac{\mathcal{A}}{R(t)} \leq-\frac{1}{2}$, since by the definition of $\chi, v_{\epsilon}^{*}=-1$ and $f_{\epsilon}=0$. In the case $r \geq \epsilon R(t)$ and $|\mathcal{A}| \geq-\epsilon \ln \epsilon$, since we are only considering $t \leq t_{*}$, (61) follows directly from (54).

Therefore, choosing $K$ (that only depends on $\gamma$ ) large enough we have

$$
\left|f_{\epsilon}(r, t)\right| \leq \epsilon,
$$

and

$$
\begin{aligned}
F_{\epsilon}(x, t) \leq & +\int_{\left\{s \leq t: R^{2}(t) \leq 6 \epsilon^{2}|\ln \epsilon|^{3+p}\right\}\{y:|y| \leq \epsilon R(t)\}} \int_{\left\{s \leq t: R^{2}(t) \geq 6 \epsilon^{2}|\ln \epsilon|^{3+p}\right\}\{y:|\mathcal{A}| \leq-K \epsilon \ln \epsilon,|y| \geq \epsilon R(t)\}} \mathcal{H}(x-y, t-s) f_{\epsilon}(y, s) d y d s \\
& +\int_{-} \mathcal{H}(x-y, t-s) f_{\epsilon}(y, s) d y d s .
\end{aligned}
$$


We are left to bound the two integrals above. Let

$$
\begin{aligned}
& I_{1}(x, t)=\int_{\left\{s \leq t: R^{2}(t) \leq 6 \epsilon^{2}|\ln \epsilon|^{3+p}\right\}} \int_{\{y: r \leq \epsilon R(t)\}} \mathcal{H}(x-y, t-s) f_{\epsilon}(y, s) d y d s \\
& I_{2}(x, t)=\int_{\left\{s \leq t: R^{2}(t) \geq 6 \epsilon^{2}|\ln \epsilon|^{3+p}\right\}\{y:|\mathcal{A}| \leq-K \epsilon \ln \epsilon,|y| \geq \epsilon R(t)\}} \mathcal{H}(x-y, t-s) f_{\epsilon}(y, s) d y d s .
\end{aligned}
$$

Before finding bounds for $I_{1}$ and $I_{2}$ recall that we only need to consider $t \leq t_{*}$, defined by (58). Now we can devote ourselves to find uniform bounds for $I_{1}$ and $I_{2}$.

- Bounds for $I_{1}$ : As mentioned before, we only need to consider $t \leq t_{*}$. From Eqs. (55), (56) and (57) we have for $r \leq \epsilon R(t)$ that

$$
\begin{aligned}
\left|\mathcal{A}_{t}\right| & \leq\left(\frac{1}{R(t)}+\frac{\epsilon^{2}|\ln \epsilon|^{3+p}}{R^{3}(t)}\right)+C\left(\frac{\epsilon^{2}}{\epsilon R(t)}+\frac{\epsilon R(t)}{\epsilon^{2}|\ln \epsilon|^{3+p}}\right) \\
\left|\mathcal{A}_{r}\right| & \leq C \\
\left|\mathcal{A}_{r r}\right| & \leq \frac{C}{\epsilon R(t)} .
\end{aligned}
$$

By definition of $v_{\epsilon}^{*}$, we need to consider only $|\mathcal{A}| \leq \frac{R(t)}{2}$ (otherwise $v_{\epsilon}^{*} \equiv 1$ and $f_{\epsilon}=0$ ). By the definition of $\mathcal{A}$, this implies that

$$
r \geq(1-\eta) r \geq \frac{R(t)}{2}-\frac{\epsilon|\ln \epsilon|^{3+p}}{R(t)} .
$$

Also by the definition of $\mathcal{A}$ we also have, when $r \leq \frac{\epsilon R(t)}{2}$ and $R^{2}(t) \leq \epsilon^{2}|\ln \epsilon|^{3+p}$, that $\mathcal{A}_{r}=\mathcal{A}_{r r}=0$. In particular this implies that we can bound $\left|\frac{\mathcal{A}_{r}}{r}\right|$ by 0 when $r \leq \frac{\epsilon R(t)}{2}$ and $R^{2}(t) \leq 3 \epsilon^{2}|\ln \epsilon|^{3+p}$. When $r \geq \frac{\epsilon R(t)}{2}$ it holds that $\left|\frac{\mathcal{A}_{r}}{r}\right| \leq \frac{C}{\epsilon R(t)}$. Finally, if $R^{2}(t) \geq 3 \epsilon^{2}|\ln \epsilon|^{3+p}$, from (64) we only need to consider $r \geq \frac{\epsilon^{2}|\ln \epsilon|^{3+p}}{2 R(t)}$ and $\left|\frac{\mathcal{A}_{r}}{r}\right| \leq \frac{C \epsilon^{2}|\ln \epsilon|^{3+p}}{R(t)}$. Hence, from Eq. (54), we have

$$
\begin{aligned}
\left|f_{\epsilon}(x, t)\right| \leq & C\left(\left[\frac{1}{R(t)}+\frac{1}{\epsilon}\right]\left[\frac{1}{R(t)}+\frac{\epsilon^{2}|\ln \epsilon|^{3+p}}{R^{3}(t)}+\frac{\epsilon}{R(t)}+\frac{R(t)}{\epsilon^{2}|\ln \epsilon|^{3+p}}+\frac{1}{\epsilon R(t)}\right]\right. \\
& \left.+\frac{1}{R^{2}(t)}+\frac{1}{R^{3}(t)}+\frac{1}{R(t) \epsilon}+\frac{1}{\epsilon^{2}}\right) .
\end{aligned}
$$

In the previous inequality using the remarks above,

Recalling that we only need to consider $t \leq t_{*}$, we have $\left|\frac{\epsilon^{2}|\ln \epsilon|^{3+p}}{R(t)}\right| \leq C$. Then we can conclude that

$$
\left|f_{\epsilon}(x, t)\right| \leq \frac{C}{\epsilon^{2} R(t)}
$$

Hence, denoting $t_{1}$ the time such that

$$
R\left(t_{1}\right)=6 \epsilon^{2}|\ln \epsilon|^{3+p},
$$


we have

$$
I_{1}(x, t) \leq \int_{t_{1}}^{t} \frac{C}{\epsilon^{2} R(s)} \int_{\{y:|r| \leq \epsilon R(s)\}} \mathcal{H}(x-y, t-s) d y d s .
$$

Fix $q>2$. Hölder's inequality implies

$$
I_{1}(x, t) \leq \int_{t_{1}}^{t} \frac{C}{\epsilon^{2} R(s)}\left(\int_{\mathbb{R}^{2}} \mathcal{H}^{q}(x-y, t-s) d y\right)^{\frac{1}{q}}\left(\int_{\{y:|r| \leq \epsilon R(s)\}} d y\right)^{\frac{1}{q^{\prime}}} d s .
$$

Using the definition of $\mathcal{H}(x-y, t-s)$, follows by integrating that

$$
I_{1}(x, t) \leq C \int_{t_{1}}^{t} \frac{(t-s)^{\frac{1}{q}-1}\left(\epsilon^{2} R^{2}(s)\right)^{\frac{1}{q^{\prime}}}}{\epsilon^{2} R(s)} d s=C \int_{t_{1}}^{t} \frac{(t-s)^{\frac{1}{q}-1}}{\epsilon^{\frac{2}{q}} R^{1-\frac{2}{q^{\prime}}}(s)} d s
$$

Notice that by definition of $R(s)$ we have $2(t-s) \leq R^{2}(s)$. Then

$$
\begin{aligned}
I_{1}(x, t) & \leq C \int_{t_{1}}^{t} \frac{1}{\epsilon^{\frac{2}{q}}(t-s)^{\frac{1}{2}}} \\
& =C \frac{\left(t-t_{1}\right)^{\frac{1}{2}}}{\epsilon^{\frac{2}{q}}} d s .
\end{aligned}
$$

Since $q>2$, by the definition of $t_{1}$ we have

$$
I_{1}(x, t) \leq C \epsilon^{1-\frac{2}{q}}|\ln \epsilon|^{\frac{3+p}{2}} \rightarrow 0 \text { as } \epsilon \rightarrow 0 .
$$

- Bounds for $I_{2}$ : As before, recall that we only need to consider $t \leq t_{*}$. In the proof we will use the two following facts:

1. For $r, t$ satisfying $|\mathcal{A}(r, t, \epsilon)| \leq-K \epsilon \ln \epsilon$, with $K$ is large enough, we have that $|\mathcal{A}(r, t, \epsilon)| \leq \frac{R(t)}{2}$ for every $t \leq t_{1}$ (where $t_{1}$ is defined by (65)) and, by the definition of $\chi, \chi\left(\frac{\mathcal{A}}{R(t)}\right) \equiv 1$. The (54) implies

$$
\begin{aligned}
\left(P v_{\epsilon}^{*}+\frac{W^{\prime}\left(v_{\epsilon}^{*}\right)}{\epsilon^{2}}\right)(x, t) & =\frac{u_{*}^{\prime}\left(\frac{\mathcal{A}}{\epsilon}\right)}{\epsilon}\left[\frac{1}{R(t)}+\frac{\epsilon^{2}|\ln \epsilon|^{3+p}}{R^{3}(t)}-\frac{1}{r}\right] \\
& =\frac{u_{*}^{\prime}\left(\frac{\mathcal{A}}{\epsilon}\right)}{\epsilon}\left[\frac{\mathcal{A}}{r R(t)}+\frac{\epsilon^{2}|\ln \epsilon|^{3+p}}{r R^{3}(t)} \mathcal{A}-\frac{\epsilon^{4}|\ln \epsilon|^{6+2 p}}{r R^{4}(t)}\right] .
\end{aligned}
$$

Noticing that when $t \leq t_{1}$ and $\mathcal{A} \geq-\frac{R(t)}{2}$, by the definition of $\mathcal{A}$ we have $r \geq \frac{R(t)}{4}$ and $R^{2}(t) \geq C \epsilon^{2}|\ln \epsilon|^{3+p}$, we can conclude using (16) and (17) that

$$
f_{\epsilon}(x, t) \leq \frac{C}{R^{2}(t)},
$$

when $t \leq t_{*}$ and $|\mathcal{A}| \leq-K \epsilon \ln \epsilon$.

2. By definition of $\mathcal{H}(x-y, t-s)$, for every $s \leq t-\epsilon^{2}|\ln \epsilon|^{3+\frac{p}{2}}$ holds that

$$
\mathcal{H}(x-y, t-s) \leq \frac{1}{\epsilon^{2}|\ln \epsilon|^{3+\frac{p}{2}}} \text {. }
$$


Which implies that

$$
\begin{aligned}
I_{2}(x, t) \leq & \frac{1}{\epsilon^{2}|\ln \epsilon|^{3+\frac{p}{2}}} \int_{0}^{t-\tau} \int_{\{y:|y| \leq-K \epsilon \ln \epsilon,|y| \geq R(s) \epsilon\}}\left|P v_{\epsilon}^{*}+\frac{W^{\prime}\left(v_{\epsilon}^{*}\right)}{\epsilon^{2}}\right| d y d s \\
& +\int_{t-\tau}^{t} \int_{\mathbb{R}^{2}} \mathcal{H}(x-y, t-s)\left|P v_{\epsilon}^{*}+\frac{W^{\prime}\left(v_{\epsilon}^{*}\right)}{\epsilon^{2}}\right| d y d s,
\end{aligned}
$$

where $\tau=\epsilon^{2}|\ln \epsilon|^{3+\frac{p}{2}}$.

Combining (67) and (68) we have that

$$
\begin{aligned}
I_{2}(x, t) \leq & \frac{1}{\epsilon^{2}|\ln \epsilon|^{3+\frac{p}{2}}} \int_{0}^{t-\tau} \int_{\{y:|y| \leq-K \epsilon \ln \epsilon,|y| \geq R(s) \epsilon\}} \frac{1}{R^{2}(s)} d y d s \\
& +\int_{t-\tau}^{t} \int_{\mathbb{R}^{2}} \mathcal{H}(x-y, t-s) \frac{1}{R^{2}(s)} d y d s \\
\leq & \frac{1}{\epsilon^{2}|\ln \epsilon|^{3+\frac{p}{2}}} \int_{0}^{t-\tau} \frac{K^{2} \epsilon^{2} \ln ^{2} \epsilon}{R^{2}(s)} d s+C \int_{t-\tau}^{t} \frac{1}{R^{2}(s)} d s .
\end{aligned}
$$

Using the definition of $R(t)$ and recalling that we are only considering $t \leq t_{*}$ we obtain

$$
\begin{aligned}
I_{2}(x, t) & \leq C \frac{K^{2} \epsilon^{2} \ln ^{2} \epsilon}{\epsilon^{2}|\ln \epsilon|^{3+\frac{p}{2}}}\left(\ln 2-\ln (2(1-t))+C \ln \left(\frac{1-t}{1-t+\tau}\right)\right. \\
& \leq C \frac{K^{2} \epsilon^{2} \ln ^{2} \epsilon}{\epsilon^{2}|\ln \epsilon|^{3+\frac{p}{2}}}\left(\ln 2-\ln \left(2\left(1-t_{*}\right)\right)+C \ln \left(\frac{1-t}{1-t+\tau}\right)\right. \\
& \leq C \frac{\left.\left|\ln \epsilon^{2}\right| \ln \epsilon\right|^{3+p} \mid}{|\ln \epsilon|^{1+\frac{p}{2}}}+C \ln \left(\frac{1}{1+\frac{\tau}{1-t}}\right) \\
& \leq C \frac{2}{|\ln \epsilon|^{\frac{p}{2}}}+C(3+p) \frac{\ln |\ln \epsilon|}{|\ln \epsilon|^{1+\frac{p}{2}}}+C \ln \left(\frac{1}{1+\frac{\tau}{1-t}}\right) .
\end{aligned}
$$

Since $t \leq t_{*}$ we have that $\frac{\tau}{1-t} \leq \frac{1}{|\ln \epsilon|^{\frac{p}{2}}} \rightarrow 0$. Therefore

$$
I_{2}(x, t) \leq C \frac{2}{|\ln \epsilon|^{\frac{p}{2}}}+C(3+p) \frac{\ln |\ln \epsilon|}{|\ln \epsilon|^{1+\frac{p}{2}}}+C \ln \left(\frac{1}{1+\frac{1}{|\ln \epsilon|^{\frac{p}{2}}}}\right) \rightarrow 0 \text { as } \epsilon \rightarrow 0
$$

Combining Eq. (66) and (68) into Eq. (63), we conclude the proof.

\section{References}

1. Ambrosio, L., Dancer, N.: Calculus of variations and partial differential equations. In: Buttazzo, G., Marino, A., Murthy, M. K. V. (eds.) Topics on Geometrical Evolution Problems and Degree Theory, Papers from the Summer School held in Pisa, September 1996, Springer, Berlin (2000) 
2. Barles, G., Soner, H.M., Souganidis, P.E.: Front propagation and phase field theory. SIAM J. Control Optim. 31(2), 439-469 (1993)

3. Bellettini, G., Paolini, M.: Quasi-optimal error estimates for the mean curvature flow with a forcing term. Diff. Integral Eqn. 8(4), 735-752 (1995)

4. Brakke, K.A.: The motion of a surface by its mean curvature, vol. 20 of Mathematical Notes. Princeton University Press, Princeton (1978)

5. Bronsard, L., Reitich, F.: On three-phase boundary motion and the singular limit of a vector-valued Ginzburg-Landau equation. Arch. Rational Mech. Anal., 124(4), 355-379 (1993)

6. Buttà, P.: Motion by mean curvature by scaling a nonlocal equation: convergence at all times in the two-dimensional case. Lett. Math. Phys., 31(1), 41-55 (1994)

7. Chen, X.: Generation and propagation of interfaces for reaction-diffusion equations. J. Differ. Eqn. 96(1), 116-141 (1992)

8. de Mottoni, P., Schatzman, M.: Geometrical evolution of developed interfaces. In Emerging applications in free boundary problems (Montreal, PQ, 1990), vol. 280 of Pitman Res. Notes Math. Ser., pp. 166-172. Longman Sci. Tech., Harlow (1993)

9. Evans, L.C., Soner, H.M., Souganidis, P.E.: Phase transitions and generalized motion by mean curvature. Comm. Pure Appl. Math., 45(9), 1097-1123 (1992)

10. Gage, M., Hamilton, R.S.: The heat equation shrinking convex plane curves. J. Differ. Geom. 23(1), 69$96(1986)$

11. Gage, M.E.: Curve shortening makes convex curves circular. Invent. Math. 76(2), 357-364 (1984)

12. Grayson, M.A.: The heat equation shrinks embedded plane curves to round points. J. Differ. Geom. 26(2), 285-314 (1987)

13. Huisken, G.: A distance comparison principle for evolving curves. Asian J. Math. 2(1), 127-133 (1998)

14. Hutchinson, J.E., Tonegawa, Y.: Convergence of phase interfaces in the van der Waals-Cahn-Hilliard theory. Calc. Var. Par. Differ. Eqn. 10(1), 49-84 (2000)

15. Ilmanen, T.: Convergence of the Allen-Cahn equation to Brakke's motion by mean curvature. J. Differ. Geom. 38(2), 417-461 (1993)

16. Mantegazza, C., Novaga, M., Tortorelli, V.M.: Motion by curvature of planar networks. Ann. Sc. Norm. Super. Pisa Cl. Sci. (5) 3(2), 235-324 (2004)

17. Nochetto, R.H., Verdi, C.: Convergence past singularities for a fully discrete approximation of curvaturedrive interfaces. SIAM J. Numer. Anal. 34(2), 490-512 (1997)

18. Pacard, F., Ritoré, M.: From constant mean curvature hypersurfaces to the gradient theory of phase transitions. J. Differ. Geom. 64(3), 359-423 (2003)

19. Paolini, M.: A quasi-optimal error estimate for a discrete singularly perturbed approximation to the prescribed curvature problem. Math. Comp. 66(217), 45-67 (1997)

20. Sáez, M.: Relaxation of the curve shortening flow via the parabolic Ginzburg-Landau equation. PhD thesis, Stanford University (2005)

21. Sternberg, P.: Vector-valued local minimizers of nonconvex variational problems. Rocky Mountain J. Math. 21(2), 799-807. Current directions in nonlinear partial differential equations (Provo, UT, 1987)(1991) 